\title{
Synthesis and Reactivity of
}

\section{Bis(alkoxyalkylidene)platinum(II) Complexes}

\author{
Michael Werner, Tadeusz Lis, Clemens Bruhn, Ronald Lindner, Dirk Steinborn ${ }^{*}$
}

\section{Supplemental Material}

Energies, Cartesian Coordinates of Atom Positions and selected Interatomic Distances in Calculated Molecules

\section{Complex 7c:}

cis-[PtCl $\left.2\{\mathrm{C}(\mathrm{OMe}) \mathrm{Me})_{2}\right]$ (carbenes: $E, E$-configuration, cisoid $)$

file: ptcl2carbene01.out

equilibrium structure

E(RB+HF-LYP)

Zero-point vibrational energies

Sum of electronic and zero-point energies $\quad-1425.948927 \mathrm{Ha}$
$-1426.12564593 \mathrm{Ha}$

$110.89304 \mathrm{kcal} / \mathrm{mol}$

$\begin{array}{llll} & x & y & z \\ \mathrm{Pt} & -0.000063 & 0.203932 & -0.135829 \\ \mathrm{Cl} & 1.757734 & 1.864356 & -0.021625 \\ \mathrm{C} & -1.520721 & -1.022126 & -0.326807 \\ \mathrm{C} & 1.521015 & -1.021681 & -0.326619 \\ \mathrm{Cl} & -1.757551 & 1.864573 & -0.022788 \\ \mathrm{O} & -2.331108 & -1.410195 & 0.609809 \\ \mathrm{C} & -1.982545 & -1.516887 & -1.663611 \\ \mathrm{O} & 2.330733 & -1.410552 & 0.610257\end{array}$




$\begin{array}{llll}\mathrm{C} & 1.984235 & -1.514646 & -1.663576 \\ \mathrm{C} & -2.164199 & -0.956083 & 1.980511 \\ \mathrm{C} & 2.162710 & -0.957542 & 1.981196 \\ \mathrm{H} & -2.816854 & -2.219965 & -1.579280 \\ \mathrm{H} & -1.153812 & -1.968512 & -2.216770 \\ \mathrm{H} & -2.299959 & -0.634761 & -2.235276 \\ \mathrm{H} & 2.816458 & -2.220196 & -1.579170 \\ \mathrm{H} & 2.305651 & -0.631832 & -2.231999 \\ \mathrm{H} & 1.155547 & -1.962216 & -2.219995 \\ \mathrm{H} & -2.937532 & -1.476654 & 2.544526 \\ \mathrm{H} & -2.299149 & 0.125934 & 2.004117 \\ \mathrm{H} & -1.167562 & -1.227286 & 2.332797 \\ \mathrm{H} & 2.936614 & -1.477332 & 2.545146 \\ \mathrm{H} & 1.166376 & -1.230547 & 2.332959 \\ \mathrm{H} & 2.296060 & 0.124657 & 2.005560\end{array}$

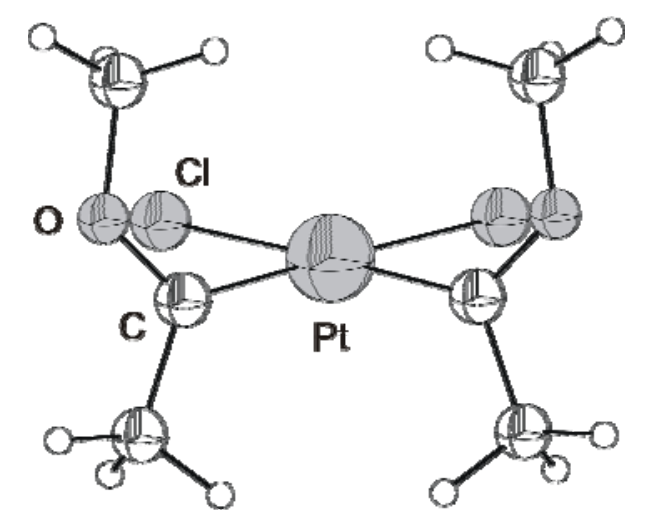

\begin{tabular}{lll}
\hline interplanar angle $^{\text {a) }}$ & $76.3^{\circ}$ & $76.2^{\circ}$ \\
$\mathrm{Pt}-\mathrm{Cl}$ & $2.421 \AA$ & $2.421 \AA$ \\
$\mathrm{Pt}-\mathrm{C}$ & $1.963 \AA$ & $1.963 \AA$ \\
$\mathrm{C}-\mathrm{O}$ & $1.298 \AA$ & $1.298 \AA$ \\
\hline
\end{tabular}

a) coordination plane $(\mathrm{C}, \mathrm{C}, \mathrm{Cl}, \mathrm{Cl})$ - ligand plane $(\mathrm{C}, \mathrm{C}, \mathrm{O}, \mathrm{C})$

\section{Complex 7ct:}


file: ptc12carbene02.out

equilibrium structure

$\mathrm{E}(\mathrm{RB}+\mathrm{HF}-\mathrm{LYP})$

Zero-point vibrational energies
$-1426.12635990 \mathrm{Ha}$

$111.00808 \mathrm{kcal} / \mathrm{mol}$

Sum of electronic and zero-point energies $\quad-1425.949457 \mathrm{Ha}$

$\begin{array}{llll} & \text { X } & y & Z \\ \mathrm{Pt} & 0.000008 & 0.197550 & 0.000028 \\ \mathrm{Cl} & 1.676217 & 1.853855 & 0.550624 \\ \mathrm{C} & -1.437915 & -1.060672 & -0.450482 \\ \mathrm{C} & 1.437956 & -1.060614 & 0.450562 \\ \mathrm{Cl} & -1.676309 & 1.853696 & -0.550819 \\ \mathrm{O} & -2.456271 & -1.348456 & 0.299453 \\ \mathrm{C} & -1.551942 & -1.731925 & -1.785566 \\ \mathrm{O} & 2.456218 & -1.348493 & -0.299472 \\ \mathrm{C} & 1.552203 & -1.731664 & 1.785722 \\ \mathrm{C} & -2.625755 & -0.720387 & 1.603155 \\ \mathrm{C} & 2.625580 & -0.720479 & -1.603231 \\ \mathrm{H} & -2.402241 & -2.419019 & -1.835170 \\ \mathrm{H} & -0.620783 & -2.253705 & -2.028102 \\ \mathrm{H} & -1.676093 & -0.936931 & -2.532574 \\ \mathrm{H} & 2.401912 & -2.419516 & 1.834961 \\ \mathrm{H} & 0.620720 & -2.252435 & 2.029116 \\ \mathrm{H} & 1.677685 & -0.936530 & 2.532378 \\ \mathrm{H} & -3.458230 & -1.251512 & 2.063569 \\ \mathrm{H} & -2.851211 & 0.334468 & 1.443314 \\ \mathrm{H} & -1.708370 & -0.827320 & 2.183776 \\ \mathrm{H} & 3.458424 & -1.251215 & -2.063422\end{array}$




$\begin{array}{llll}\mathrm{H} & 2.850429 & 0.334523 & -1.443513 \\ \mathrm{H} & 1.708356 & -0.828057 & -2.183987\end{array}$

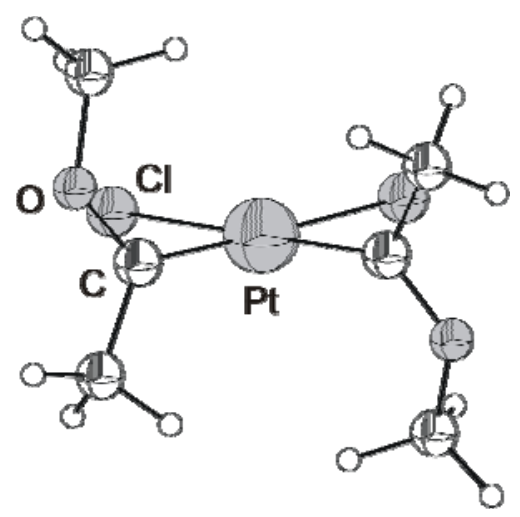

\begin{tabular}{lll}
\hline interplanar angle $^{\mathrm{a})}$ & $77.3^{\circ}$ & $77.3^{\circ}$ \\
$\mathrm{Pt}-\mathrm{Cl}$ & $2.420 \AA$ & $2.420 \AA$ \\
$\mathrm{Pt}-\mathrm{C}$ & $1.963 \AA$ & $1.963 \AA$ \\
$\mathrm{C}-\mathrm{O}$ & $1.297 \AA$ & $1.297 \AA$
\end{tabular}

a) coordination plane $(\mathrm{C}, \mathrm{C}, \mathrm{Cl}, \mathrm{Cl})$ - ligand plane $(\mathrm{C}, \mathrm{C}, \mathrm{O}, \mathrm{C})$

Transition state ts2 $\left(7_{\mathrm{ct}} \rightarrow 7_{\mathrm{cc}}\right)$ :

cis-[PtCl $\left.{ }_{2}\{\mathrm{C}(\mathrm{OMe}) \mathrm{Me})_{2}\right]$ (carbenes: $E, E$-configuration)

file: ptc12carbene24_exoexo_ts.out

transition structure (carbenes: transoid $\longleftarrow \longrightarrow$ cisoid)

$\mathrm{E}(\mathrm{RB}+\mathrm{HF}-\mathrm{LYP}) \quad-1426.11263909 \mathrm{Ha}$

Zero-point vibrational energies $\quad 111.34151 \mathrm{kcal} / \mathrm{mol}$

Sum of electronic and zero-point Energies $\quad-1425.935205 \mathrm{Ha}$

$\begin{array}{llll} & x & y & Z \\ \text { Pt } & 0.040583 & -0.206578 & -0.084645 \\ \text { Cl } & -1.635700 & -1.967562 & -0.143944 \\ \text { C } & 1.213459 & 1.383348 & -0.025910 \\ \text { C } & -1.614833 & 0.831368 & -0.321886\end{array}$




\begin{tabular}{|c|c|c|c|}
\hline $\mathrm{Cl}$ & 1.691515 & -1.929435 & 0.097299 \\
\hline $\mathrm{O}$ & 2.498517 & 1.537128 & 0.105212 \\
\hline $\mathrm{C}$ & 0.625778 & 2.778615 & -0.134223 \\
\hline $\mathrm{O}$ & -2.446160 & 1.175549 & 0.615169 \\
\hline $\mathrm{C}$ & -2.168398 & 1.161170 & -1.675191 \\
\hline $\mathrm{C}$ & 3.516033 & 0.509346 & 0.236385 \\
\hline $\mathrm{C}$ & -2.204744 & 0.790702 & 1.996795 \\
\hline $\mathrm{H}$ & 1.397506 & 3.547212 & -0.039395 \\
\hline $\mathrm{H}$ & -0.134271 & 2.927239 & 0.640050 \\
\hline $\mathrm{H}$ & 0.126213 & 2.890275 & -1.102960 \\
\hline $\mathrm{H}$ & -3.000813 & 1.869815 & -1.620258 \\
\hline $\mathrm{H}$ & -2.531922 & 0.212228 & -2.092187 \\
\hline $\mathrm{H}$ & -1.385130 & 1.520828 & -2.346888 \\
\hline $\mathrm{H}$ & 4.444363 & 1.075339 & 0.320056 \\
\hline $\mathrm{H}$ & 3.506226 & -0.130444 & -0.643143 \\
\hline $\mathrm{H}$ & 3.321777 & -0.088212 & 1.124411 \\
\hline $\mathrm{H}$ & -2.947486 & 1.337280 & 2.577079 \\
\hline $\mathrm{H}$ & -1.187847 & 1.060300 & 2.287046 \\
\hline $\mathrm{H}$ & -2.345536 & -0.288519 & 2.072622 \\
\hline
\end{tabular}

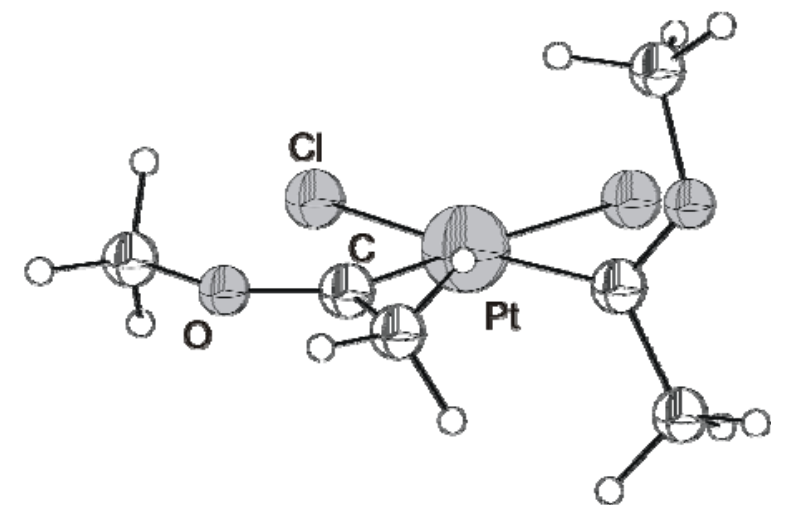

\begin{tabular}{lll}
\hline interplanar angle $^{\text {a) }}$ & $1.6^{\circ}$ & $84.9^{\circ}$ \\
$\mathrm{Pt}-\mathrm{Cl}$ & $2.393 \AA$ & $2.432 \AA$ \\
\hline
\end{tabular}




\begin{tabular}{lll}
\hline $\mathrm{Pt}-\mathrm{C}$ & $1.977 \AA$ & $1.968 \AA$ \\
$\mathrm{C}-\mathrm{O}$ & $1.301 \AA$ & $1.299 \AA$ \\
\hline
\end{tabular}

a) coordination plane $(\mathrm{C}, \mathrm{C}, \mathrm{Cl}, \mathrm{Cl})$ - ligand plane $(\mathrm{C}, \mathrm{C}, \mathrm{O}, \mathrm{C})$

Transition state ts1 $\left(7_{\mathrm{ct}} \rightarrow 7_{\mathrm{cc}}\right)$ :

cis-[ $\left.\mathrm{PtCl}_{2}\{\mathrm{C}(\mathrm{OMe}) \mathrm{Me})_{2}\right]$ (carbenes: $E, E$-configuration)

file: ptcl2carbene23_exoexo_ts.out

transition structure (carbenes: transoid $\longleftrightarrow \longrightarrow$ cisoid)

$\mathrm{E}(\mathrm{RB}+\mathrm{HF}-\mathrm{LYP})$

Zero-point vibrational energies

Sum of electronic and zero-point energies $\quad-1425.934779 \mathrm{Ha}$
$\begin{array}{llll} & x & y & z \\ \text { Pt } & 0.217157 & -0.235180 & -0.069975\end{array}$
$\begin{array}{llll}\mathrm{Cl} & -0.949973 & -2.358538 & -0.155496\end{array}$
$\begin{array}{llll}\text { C } & 1.195287 & 1.475845 & 0.050180\end{array}$
$\begin{array}{llll}\text { C } & -1.648700 & 0.327400 & -0.327400\end{array}$
$\begin{array}{llll}\mathrm{Cl} & 2.267771 & -1.461932 & 0.110976\end{array}$
$\begin{array}{llll}\mathrm{O} & 0.748728 & 2.711251 & -0.007123\end{array}$
$\begin{array}{llll}\text { C } & 2.681087 & 1.561696 & 0.254329\end{array}$
$\begin{array}{llll}\text { O } & -2.561119 & 0.429000 & 0.596939\end{array}$
$\begin{array}{llll}\text { C } & -2.267807 & 0.443461 & -1.690829\end{array}$
$\begin{array}{llll}\text { C } & -0.621472 & 3.099683 & -0.191700\end{array}$
$\begin{array}{llll}\text { C } & -2.236877 & 0.147562 & 1.985050\end{array}$
$\begin{array}{llll}\mathrm{H} & 3.006262 & 2.604404 & 0.306089\end{array}$
$\begin{array}{llll}\mathrm{H} & 3.188753 & 1.031109 & -0.556612\end{array}$
$\begin{array}{llll}\mathrm{H} & 2.956303 & 1.019426 & 1.163608\end{array}$
$\begin{array}{llll}\mathrm{H} & -3.224153 & 0.976452 & -1.665969\end{array}$
$\begin{array}{llll}\mathrm{H} & -2.449529 & -0.587494 & -2.023401\end{array}$ 


$\begin{array}{llll}\mathrm{H} & -1.578262 & 0.892216 & -2.408841 \\ \mathrm{H} & -0.612168 & 4.188618 & -0.139206 \\ \mathrm{H} & -1.245327 & 2.693478 & 0.604365 \\ \mathrm{H} & -0.975134 & 2.778869 & -1.171878 \\ \mathrm{H} & -3.104595 & 0.480647 & 2.553911 \\ \mathrm{H} & -1.332458 & 0.686253 & 2.275038 \\ \mathrm{H} & -2.080463 & -0.927862 & 2.081482\end{array}$

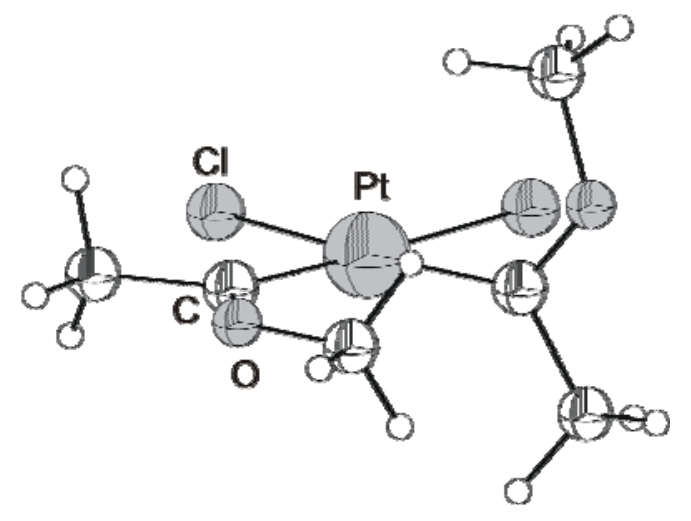

\begin{tabular}{lll}
\hline interplanar angle $^{\text {a) }}$ & $1.5^{\circ}$ & $86.4^{\circ}$ \\
$\mathrm{Pt}-\mathrm{Cl}$ & $2.396 \AA$ & $2.424 \AA$ \\
$\mathrm{Pt}-\mathrm{C}$ & $1.975 \AA$ & $1.966 \AA$ \\
$\mathrm{C}-\mathrm{O}$ & $1.315 \AA$ & $1.303 \AA$ \\
\hline
\end{tabular}

a) coordination plane $(\mathrm{C}, \mathrm{C}, \mathrm{Cl}, \mathrm{Cl})$ - ligand plane $(\mathrm{C}, \mathrm{C}, \mathrm{O}, \mathrm{C})$

\section{Complex 7t:}

trans-[PtCl$\left.{ }_{2}\{\mathrm{C}(\mathrm{OMe}) \mathrm{Me})_{2}\right]$ (carbenes: $E, E$-configuration, cisoid)

file: ptc12carbene44trans_cis.out

equilibrium structure

$\mathrm{E}(\mathrm{RB}+\mathrm{HF}-\mathrm{LYP})$

Zero-point vibrational energies

Sum of electronic and zero-point energies $\quad-1425.946873 \mathrm{Ha}$
$-1426.12323138 \mathrm{Ha}$

$110.66638 \mathrm{kcal} / \mathrm{mol}$ 


\begin{tabular}{|c|c|c|c|}
\hline & $x$ & $y$ & $Z$ \\
\hline $\mathrm{Pt}$ & 0.000043 & 0.000157 & -0.149653 \\
\hline $\mathrm{C}$ & 2.006961 & -0.272809 & -0.238119 \\
\hline $\mathrm{C}$ & -2.006957 & 0.272874 & -0.237866 \\
\hline $\mathrm{O}$ & 2.885663 & -0.011711 & 0.677493 \\
\hline $\mathrm{C}$ & 2.652998 & -0.825904 & -1.464922 \\
\hline $\mathrm{O}$ & -2.885635 & 0.010719 & 0.677454 \\
\hline $\mathrm{C}$ & -2.653088 & 0.826630 & -1.464319 \\
\hline $\mathrm{C}$ & 2.459119 & 0.512291 & 1.967607 \\
\hline $\mathrm{C}$ & -2.459154 & -0.514209 & 1.967186 \\
\hline $\mathrm{H}$ & 3.740683 & -0.908472 & -1.374597 \\
\hline $\mathrm{H}$ & 2.386278 & -0.186602 & -2.315568 \\
\hline $\mathrm{H}$ & 2.203927 & -1.807312 & -1.664036 \\
\hline $\mathrm{H}$ & -3.740823 & 0.908631 & -1.374091 \\
\hline $\mathrm{H}$ & -2.385937 & 0.188146 & -2.315441 \\
\hline $\mathrm{H}$ & -2.204437 & 1.808406 & -1.662581 \\
\hline $\mathrm{H}$ & 3.356936 & 0.506676 & 2.585259 \\
\hline $\mathrm{H}$ & 1.689062 & -0.140823 & 2.380351 \\
\hline $\mathrm{H}$ & 2.073851 & 1.521873 & 1.825293 \\
\hline $\mathrm{H}$ & -3.355410 & -0.502461 & 2.587017 \\
\hline $\mathrm{H}$ & -1.683918 & 0.134411 & 2.377276 \\
\hline $\mathrm{H}$ & -2.080454 & -1.526293 & 1.825003 \\
\hline $\mathrm{Cl}$ & 0.306048 & 2.369037 & -0.152869 \\
\hline $\mathrm{Cl}$ & -0.306200 & -2.368667 & -0.154069 \\
\hline
\end{tabular}




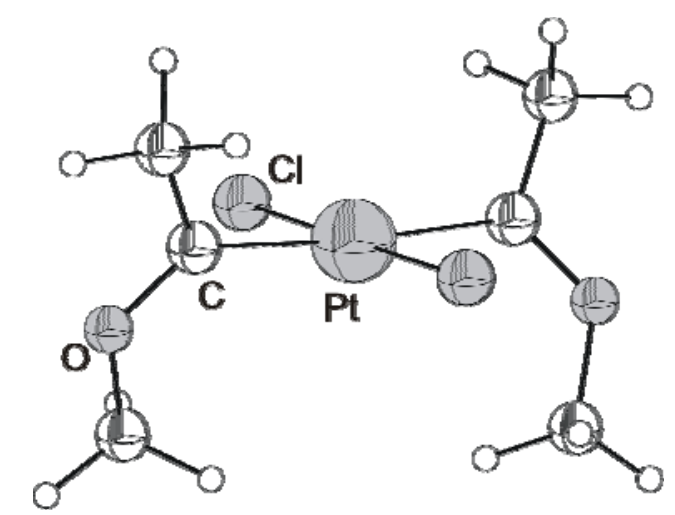

\begin{tabular}{lll}
\hline interplanar angle $^{\text {a) }}$ & $69.1^{\circ}$ & $69.1^{\circ}$ \\
$\mathrm{Pt}-\mathrm{Cl}$ & $2.389 \AA$ & $2.389 \AA$ \\
$\mathrm{Pt}-\mathrm{C}$ & $2.027 \AA$ & $2.027 \AA$ \\
$\mathrm{C}-\mathrm{O}$ & $1.296 \AA$ & $1.296 \AA$
\end{tabular}

a) coordination plane $(\mathrm{C}, \mathrm{C}, \mathrm{Cl}, \mathrm{Cl})$ - ligand plane $(\mathrm{C}, \mathrm{C}, \mathrm{O}, \mathrm{C})$

\section{Complex $7_{\mathrm{tt}}$ :}

trans-[PtCl $\left.{ }_{2}\{\mathrm{C}(\mathrm{OMe}) \mathrm{Me})_{2}\right]$ (carbenes: E,E-configuration, transoid)

file: ptc12carbene42trans_trans.out

equilibrium structure

$\mathrm{E}(\mathrm{RB}+\mathrm{HF}-\mathrm{LYP})$

Zero-point vibrational energies

Sum of electronic and zero-point energies $\quad-1425.946650 \mathrm{Ha}$
$-1426.12284732 \mathrm{Ha}$

$110.56562 \mathrm{kcal} / \mathrm{mol}$

$\begin{array}{llll} & x & y & Z \\ \text { Pt } & -0.000181 & 0.000807 & -0.000895 \\ \text { C } & -1.942938 & 0.416595 & 0.408403 \\ \text { C } & 1.942694 & -0.414320 & -0.410643 \\ \text { O } & -2.979480 & -0.004313 & -0.243205 \\ \text { C } & -2.338868 & 1.291388 & 1.550930 \\ \text { O } & 2.978070 & -0.003905 & 0.249280 \\ \text { C } & 2.339759 & -1.274835 & -1.563504\end{array}$




$\begin{array}{llll}\mathrm{C} & -2.799466 & -0.859582 & -1.410380 \\ \mathrm{C} & 2.795422 & 0.838120 & 1.425652 \\ \mathrm{H} & -3.422987 & 1.410184 & 1.644455 \\ \mathrm{H} & -1.855299 & 2.265925 & 1.405896 \\ \mathrm{H} & -1.918591 & 0.869973 & 2.472413 \\ \mathrm{H} & 3.423850 & -1.395109 & -1.655536 \\ \mathrm{H} & 1.923286 & -0.839633 & -2.480306 \\ \mathrm{H} & 1.853445 & -2.250058 & -1.433242 \\ \mathrm{H} & -3.807821 & -1.051136 & -1.776541 \\ \mathrm{H} & -2.302066 & -1.778769 & -1.100862 \\ \mathrm{H} & -2.203888 & -0.325399 & -2.151736 \\ \mathrm{H} & 3.802856 & 1.024904 & 1.796777 \\ \mathrm{H} & 2.197369 & 0.295909 & 2.159133 \\ \mathrm{H} & 2.299613 & 1.761190 & 1.125118 \\ \mathrm{Cl} & 0.281819 & 2.210606 & -0.861090 \\ \mathrm{Cl} & -0.278523 & -2.208803 & 0.861849\end{array}$

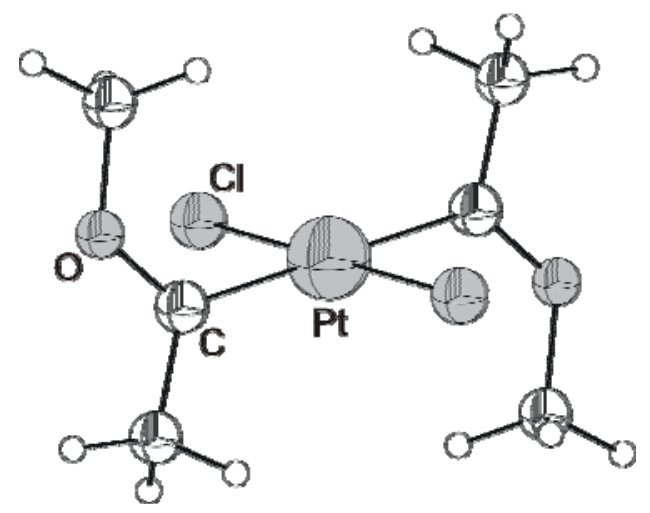

\begin{tabular}{lll}
\hline interplanar angle $^{\text {a) }}$ & $74.5^{\circ}$ & $75.2^{\circ}$ \\
$\mathrm{Pt}-\mathrm{Cl}$ & $2.388 \AA$ & $2.388 \AA$ \\
$\mathrm{Pt}-\mathrm{C}$ & $2.028 \AA$ & $2.029 \AA$ \\
$\mathrm{C}-\mathrm{O}$ & $1.295 \AA$ & $1.295 \AA$
\end{tabular}

a) coordination plane $(\mathrm{C}, \mathrm{C}, \mathrm{Cl}, \mathrm{Cl})-$ ligand plane $(\mathrm{C}, \mathrm{C}, \mathrm{O}, \mathrm{C})$ 
Transition state $\mathbf{t s}\left(7_{\mathrm{tt}} \rightarrow 7_{\mathrm{tc}}\right)$ :

trans-[PtCl$\left.{ }_{2}\{\mathrm{C}(\mathrm{OMe}) \mathrm{Me})_{2}\right]$ (carbenes: $E, E$-configuration)

file: ptc12carbene43trans_ts.out

transition structure (carbenes: transoid $\longleftarrow \longrightarrow$ cisoid)

$\mathrm{E}(\mathrm{RB}+\mathrm{HF}-\mathrm{LYP})$

Zero-point vibrational energies

Sum of electronic and zero-point energies $\quad-1425.934891 \mathrm{Ha}$
$-1426.11177967 \mathrm{Ha}$

$110.99927 \mathrm{kcal} / \mathrm{mol}$

$\begin{array}{lll}x & y & Z\end{array}$

Pt $\quad 0.017146 \quad 0.062588 \quad-0.085917$

$\begin{array}{llll}\text { C } & 2.011288 & 0.573934 & 0.087139\end{array}$

C $\quad-1.956303 \quad-0.288043 \quad-0.348634$

O $\quad 3.096577 \quad-0.141647 \quad 0.159356$

$\begin{array}{llll}\text { C } & 2.433833 & 2.016354 & 0.125331\end{array}$

$\begin{array}{llll}\text { O } & -2.844059 & -0.410942 & 0.580757\end{array}$

$\begin{array}{llll}\text { C } & -2.548795 & -0.421680 & -1.710769\end{array}$

$\begin{array}{llll}\text { C } & 3.140689 & -1.590359 & 0.152123\end{array}$

$\begin{array}{llll}\text { C } & -2.441628 & -0.301947 & 1.977984\end{array}$

$\begin{array}{llll}\mathrm{H} & 3.520385 & 2.114517 & 0.204367\end{array}$

$\begin{array}{llll}\mathrm{H} & 1.937407 & 2.516544 & 0.963467\end{array}$

$\mathrm{H} \quad 2.063283 \quad 2.524605 \quad-0.771062$

$\mathrm{H} \quad-3.626868 \quad-0.608643 \quad-1.684911$

$\mathrm{H} \quad-2.029367 \quad-1.235684 \quad-2.230076$

$\mathrm{H} \quad-2.332958 \quad 0.499118 \quad-2.266150$

$\begin{array}{llll}\mathrm{H} & 4.202587 & -1.828152 & 0.220497\end{array}$

$\mathrm{H} \quad 2.706223 \quad-1.967839 \quad-0.771706$

H $2.589796 \quad-1.979953 \quad 1.006266$

$\begin{array}{llll}\mathrm{H} & -3.361718 & -0.432378 & 2.546863\end{array}$

$\begin{array}{llll}\mathrm{H} & -2.006959 & 0.684846 & 2.141054\end{array}$ 


$\begin{array}{llll}\mathrm{H} & -1.721777 & -1.091206 & 2.197884 \\ \mathrm{Cl} & -0.661200 & 2.362170 & -0.070575 \\ \mathrm{Cl} & 0.124021 & -2.337846 & -0.075008\end{array}$

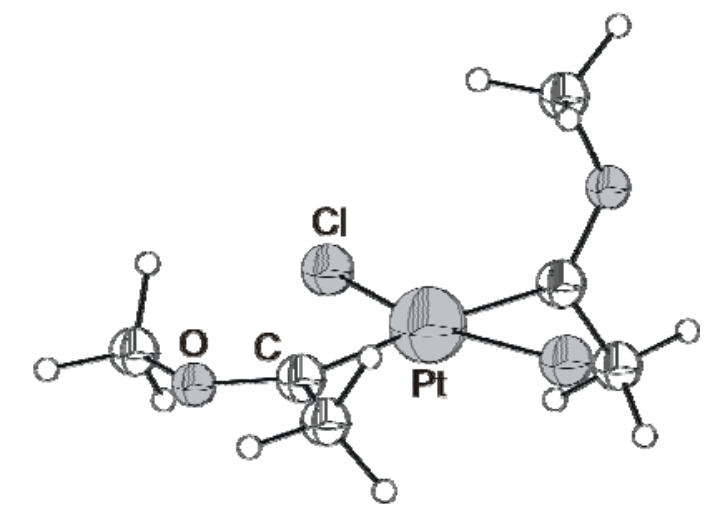

\begin{tabular}{lll}
\hline interplanar angle $^{\text {a) }}$ & $2.0^{\circ}$ & $88.4^{\circ}$ \\
$\mathrm{Pt}-\mathrm{Cl}$ & $2.398 \AA$ & $2.403 \AA$ \\
$\mathrm{Pt}-\mathrm{C}$ & $2.066 \AA$ & $2.022 \AA$ \\
$\mathrm{C}-\mathrm{O}$ & $1.302 \AA$ & $1.291 \AA$
\end{tabular}

a) coordination plane $(\mathrm{C}, \mathrm{C}, \mathrm{Cl}, \mathrm{Cl})$ - ligand plane $(\mathrm{C}, \mathrm{C}, \mathrm{O}, \mathrm{C})$

\section{Complex 8c:}

cis-[PtBr 2 (C(OMe)Me $\left.)_{2}\right]$ (carbenes: $E, E$-configuration, cisoid)

file: ptbr2carbene01.out

equilibrium structure

$\mathrm{E}(\mathrm{RB}+\mathrm{HF}-\mathrm{LYP})$

Zero-point vibrational energies
$-5648.56488563 \mathrm{Ha}$

$110.48065 \mathrm{kcal} / \mathrm{mol}$

Sum of electronic and zero-point Energies $\quad-5648.388824 \mathrm{Ha}$

$\begin{array}{llll} & x & y & Z \\ \mathrm{Pt} & -0.000041 & 0.125610 & -0.130042 \\ \mathrm{Br} & -1.869804 & -1.601569 & 0.023705 \\ \mathrm{C} & 1.531527 & 1.338075 & -0.362829\end{array}$




\begin{tabular}{|c|c|c|c|c|c|}
\hline $\mathrm{C}$ & -1.531551 & 1.338078 & -0.362872 & & \\
\hline $\mathrm{Br}$ & 1.869886 & -1.601412 & 0.023494 & & \\
\hline $\mathrm{O}$ & 2.322073 & 1.799850 & 0.558744 & & \\
\hline $\mathrm{C}$ & 1.997232 & 1.766804 & -1.722531 & & \\
\hline $\mathrm{O}$ & -2.321963 & 1.800082 & 0.558720 & & \\
\hline $\mathrm{C}$ & -1.997532 & 1.766431 & -1.722591 & & \\
\hline $\mathrm{C}$ & 2.148691 & 1.425012 & 1.950116 & & \\
\hline $\mathrm{C}$ & -2.148382 & 1.425436 & 1.950140 & & \\
\hline $\mathrm{H}$ & 2.861591 & 2.435777 & -1.669699 & & \\
\hline $\mathrm{H}$ & 1.178646 & 2.245335 & -2.270023 & & \\
\hline $\mathrm{H}$ & 2.261957 & 0.859416 & -2.278866 & & \\
\hline $\mathrm{H}$ & 15 & 0 & -2.861066 & 2.436466 & -1.669724 \\
\hline $\mathrm{H}$ & -2.263851 & 0.858862 & -2.277930 & & \\
\hline $\mathrm{H}$ & -1.178746 & 2.243357 & -2.271119 & & \\
\hline $\mathrm{H}$ & 2.892022 & 2.007188 & 2.493970 & & \\
\hline $\mathrm{H}$ & 2.325039 & 0.351907 & 2.039818 & & \\
\hline $\mathrm{H}$ & 1.136590 & 1.674642 & 2.272343 & & \\
\hline $\mathrm{H}$ & -2.892968 & 2.006237 & 2.493743 & & \\
\hline $\mathrm{H}$ & -1.136882 & 1.677095 & 2.272701 & & \\
\hline $\mathrm{H}$ & -2.322784 & 0.352015 & 2.039803 & & \\
\hline
\end{tabular}

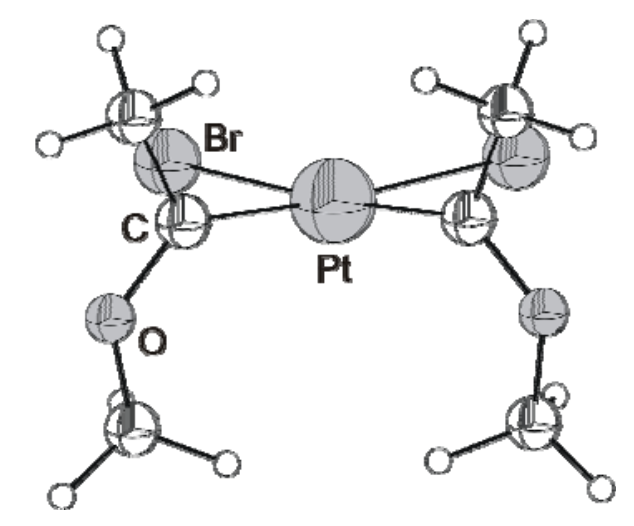

interplanar angle $^{\text {a) }} \quad 78.3^{\circ} \quad 78.3^{\circ}$ 


\begin{tabular}{lll}
\hline $\mathrm{Pt}-\mathrm{Br}$ & $2.550 \AA$ & $2.550 \AA$ \\
$\mathrm{Pt}-\mathrm{C}$ & $1.967 \AA$ & $1.967 \AA$ \\
$\mathrm{C}-\mathrm{O}$ & $1.299 \AA$ & $1.299 \AA$ \\
\hline
\end{tabular}

a) coordination plane $(\mathrm{C}, \mathrm{C}, \mathrm{Cl}, \mathrm{Cl})$ - ligand plane $(\mathrm{C}, \mathrm{C}, \mathrm{O}, \mathrm{C})$

\section{Complex 8ct:}

cis-[ $\left.\mathrm{PtBr}_{2}\{\mathrm{C}(\mathrm{OMe}) \mathrm{Me})_{2}\right]$ (carbenes: $E, E$-configuration, transoid)

file: ptbr2carbene02.out

equilibrium structure

$\mathrm{E}(\mathrm{RB}+\mathrm{HF}-\mathrm{LYP})$

Zero-point vibrational energies

Sum of electronic and zero-point energies $\quad-5648.389449 \mathrm{Ha}$
$-5648.56553099 \mathrm{Ha}$

$110.49293 \mathrm{kcal} / \mathrm{mol}$ $\begin{array}{lll}x & y & z\end{array}$

$\begin{array}{llll}\mathrm{Pt} & -0.000012 & 0.134619 & 0.000003\end{array}$

$\begin{array}{llll}\text { C } & 1.500678 & 1.373046 & -0.288645\end{array}$

$\begin{array}{llll}\text { C } & -1.500706 & 1.373032 & 0.288666\end{array}$

$\begin{array}{llll}\text { O } & 2.392285 & 1.712783 & 0.592235\end{array}$

$\begin{array}{llll}\text { C } & 1.794063 & 2.007818 & -1.615779\end{array}$

$\begin{array}{llll}\text { O } & -2.392304 & 1.712795 & -0.592214\end{array}$

$\begin{array}{llll}\text { C } & -1.794076 & 2.007807 & 1.615802\end{array}$

$\begin{array}{llll}\text { C } & 2.375661 & 1.142547 & 1.929941\end{array}$

$\begin{array}{llll}\text { C } & -2.375672 & 1.142596 & -1.929937\end{array}$

$\begin{array}{llll}\mathrm{H} & 2.732091 & 2.571185 & -1.605258\end{array}$

$\begin{array}{llll}\mathrm{H} & 0.964440 & 2.669984 & -1.891078\end{array}$

$\mathrm{H} \quad 1.838096 \quad 1.214858 \quad-2.370271$

$\begin{array}{llll}\mathrm{H} & -2.732041 & 2.571277 & 1.605255\end{array}$

$\begin{array}{llll}\mathrm{H} & -0.964388 & 2.669860 & 1.891172\end{array}$

$\begin{array}{llll}\mathrm{H} & -1.838240 & 1.214822 & 2.370263\end{array}$ 


$\begin{array}{llll}\mathrm{H} & 3.102964 & 1.725634 & 2.494206 \\ \mathrm{H} & 2.667838 & 0.094287 & 1.850357 \\ \mathrm{H} & 1.374455 & 1.223700 & 2.355387 \\ \mathrm{H} & -3.103108 & 1.725578 & -2.494138 \\ \mathrm{H} & -2.667663 & 0.094283 & -1.850371 \\ \mathrm{H} & -1.374509 & 1.223944 & -2.355448 \\ \mathrm{Br} & 1.842318 & -1.588330 & -0.375291 \\ \mathrm{Br} & -1.842276 & -1.588396 & 0.375270\end{array}$

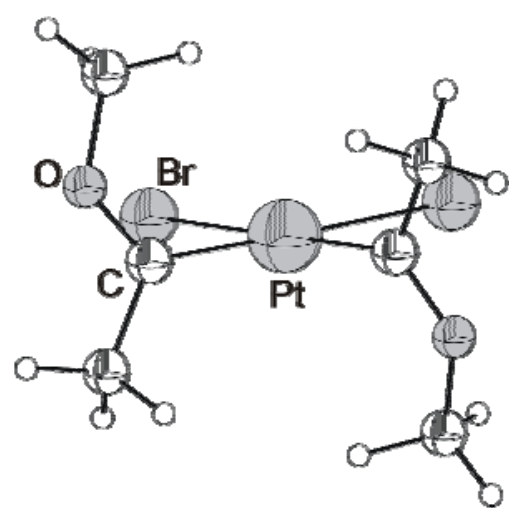

\begin{tabular}{lll}
\hline interplanar angle $^{\text {a) }}$ & $80.4^{\circ}$ & $80.4^{\circ}$ \\
$\mathrm{Pt}-\mathrm{Br}$ & $2.550 \AA$ & $2.550 \AA$ \\
$\mathrm{Pt}-\mathrm{C}$ & $1.967 \AA$ & $1.967 \AA$ \\
$\mathrm{C}-\mathrm{O}$ & $1.299 \AA$ & $1.299 \AA$
\end{tabular}

a) coordination plane $(\mathrm{C}, \mathrm{C}, \mathrm{Cl}, \mathrm{Cl})$ - ligand plane $(\mathrm{C}, \mathrm{C}, \mathrm{O}, \mathrm{C})$

Transition state $\operatorname{ts} 1\left(8_{\mathrm{ct}} \rightarrow \mathbf{8}_{\mathrm{cc}}\right)$ cis-[PtBr 2 $\left.\{\mathrm{C}(\mathrm{OMe}) \mathrm{Me})_{2}\right]$ (carbenes: $E, E$-configuration)

file: ptbr2carbene05_ts.out

transition structure (carbenes: transoid $\longleftarrow \longrightarrow$ cisoid)

$\mathrm{E}(\mathrm{RB}+\mathrm{HF}-\mathrm{LYP})$

Zero-point vibrational energies

Sum of electronic and zero-point energies $\quad-5648.371712 \mathrm{Ha}$ 


\begin{tabular}{|c|c|c|c|}
\hline & $x$ & $y$ & $Z$ \\
\hline $\mathrm{Pt}$ & 0.037418 & 0.148873 & -0.070203 \\
\hline $\mathrm{Br}$ & 0.004302 & -2.404395 & -0.090741 \\
\hline $\mathrm{C}$ & 0.070378 & 2.131226 & 0.009912 \\
\hline $\mathrm{C}$ & -1.877931 & -0.254594 & -0.311683 \\
\hline $\mathrm{Br}$ & 2.559676 & 0.025827 & 0.083090 \\
\hline $\mathrm{O}$ & -0.924722 & 2.989518 & -0.043100 \\
\hline $\mathrm{C}$ & 1.323577 & 2.943527 & 0.175766 \\
\hline $\mathrm{O}$ & -2.740956 & -0.550200 & 0.620346 \\
\hline $\mathrm{C}$ & -2.489565 & -0.459621 & -1.671174 \\
\hline $\mathrm{C}$ & -2.315632 & 2.662242 & -0.186218 \\
\hline $\mathrm{C}$ & -2.330531 & -0.610856 & 2.011257 \\
\hline $\mathrm{H}$ & 1.093078 & 4.012412 & 0.195727 \\
\hline $\mathrm{H}$ & 2.021462 & 2.705154 & -0.631823 \\
\hline $\mathrm{H}$ & 1.834532 & 2.637383 & 1.093297 \\
\hline $\mathrm{H}$ & -3.582049 & -0.386639 & -1.641340 \\
\hline $\mathrm{H}$ & -2.216309 & -1.476247 & -1.980982 \\
\hline $\mathrm{H}$ & -2.064579 & 0.225225 & -2.408195 \\
\hline $\mathrm{H}$ & -2.828894 & 3.624165 & -0.185601 \\
\hline $\mathrm{H}$ & -2.653439 & 2.059627 & 0.656790 \\
\hline $\mathrm{H}$ & -2.490039 & 2.149913 & -1.132516 \\
\hline $\mathrm{H}$ & -3.257551 & -0.659154 & 2.582271 \\
\hline $\mathrm{H}$ & -1.741328 & 0.272184 & 2.267728 \\
\hline $\mathrm{H}$ & -1.729079 & -1.512330 & 2.143113 \\
\hline
\end{tabular}




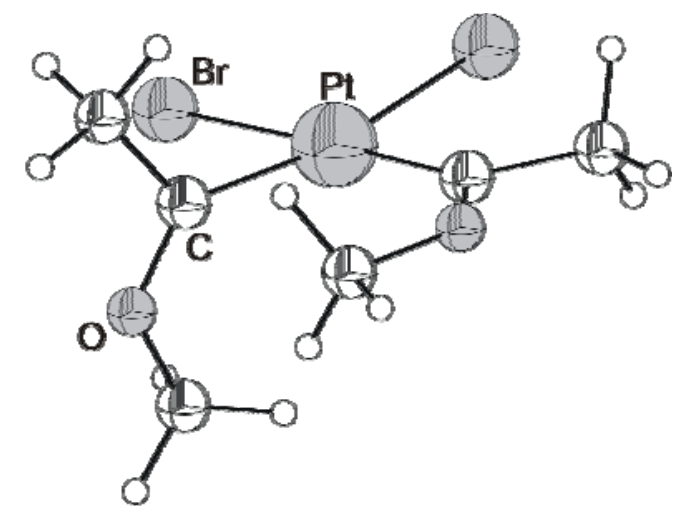

\begin{tabular}{lll}
\hline interplanar angle $^{\text {a) }}$ & $86.8^{\circ}$ & $1.9^{\circ}$ \\
$\mathrm{Pt}-\mathrm{Br}$ & $2.554 \AA$ & $2.530 \AA$ \\
$\mathrm{Pt}-\mathrm{C}$ & $1.972 \AA$ & $1.984 \AA$ \\
$\mathrm{C}-\mathrm{O}$ & $1.304 \AA$ & $1.315 \AA$
\end{tabular}

a) coordination plane $(\mathrm{C}, \mathrm{C}, \mathrm{Cl}, \mathrm{Cl})$ - ligand plane $(\mathrm{C}, \mathrm{C}, \mathrm{O}, \mathrm{C})$

Transition state ts $2\left(8_{\mathrm{ct}} \rightarrow \mathbf{8}_{\mathrm{cc}}\right)$

cis-[PtBr $\left.2\{\mathrm{C}(\mathrm{OMe}) \mathrm{Me})_{2}\right]$ (carbenes: $E, E$-configuration)

file: ptbr2carbene11ts.out

transition structure (carbenes: transoid $\longleftarrow \longrightarrow$ cisoid)

$\mathrm{E}(\mathrm{RB}+\mathrm{HF}-\mathrm{LYP})$

Zero-point vibrational energies

Sum of electronic and zero-point energies $\quad-5648.371462 \mathrm{Ha}$
$-5648.54798703 \mathrm{Ha}$

$110.77084 \mathrm{kcal} / \mathrm{mol}$

$\begin{array}{llll} & x & y & z \\ \mathrm{Pt} & 0.043598 & 0.144607 & -0.082530 \\ \mathrm{Br} & -1.743838 & -1.694067 & -0.084766 \\ \mathrm{C} & 1.200454 & 1.757242 & -0.064576 \\ \mathrm{C} & 1.628601 & 1.173584 & -0.307154 \\ \mathrm{Br} & 1.774938 & -1.687599 & 0.089072 \\ \mathrm{O} & 2.483636 & 1.944509 & 0.041242 \\ \mathrm{C} & 0.582321 & 3.139731 & -0.177481\end{array}$




$\begin{array}{llll}\mathrm{O} & -2.429553 & 1.567588 & 0.638615 \\ \mathrm{C} & -2.201793 & 1.490430 & -1.657959 \\ \mathrm{C} & 3.529260 & 0.946882 & 0.164019 \\ \mathrm{C} & -2.166546 & 1.217725 & 2.023752 \\ \mathrm{H} & 1.341971 & 3.923735 & -0.117045 \\ \mathrm{H} & -0.156469 & 3.286980 & 0.617288 \\ \mathrm{H} & 0.052169 & 3.227161 & -1.132040 \\ \mathrm{H} & -3.025282 & 2.208930 & -1.595793 \\ \mathrm{H} & -2.579884 & 0.544484 & -2.065517 \\ \mathrm{H} & -1.424229 & 1.839663 & -2.342236 \\ \mathrm{H} & 4.444683 & 1.537181 & 0.219244 \\ \mathrm{H} & 3.515990 & 0.293702 & -0.705616 \\ \mathrm{H} & 3.369850 & 0.357006 & 1.064244 \\ \mathrm{H} & -2.870329 & 1.811506 & 2.606331 \\ \mathrm{H} & -1.131448 & 1.450803 & 2.279580 \\ \mathrm{H} & -2.349405 & 0.147497 & 2.135725\end{array}$

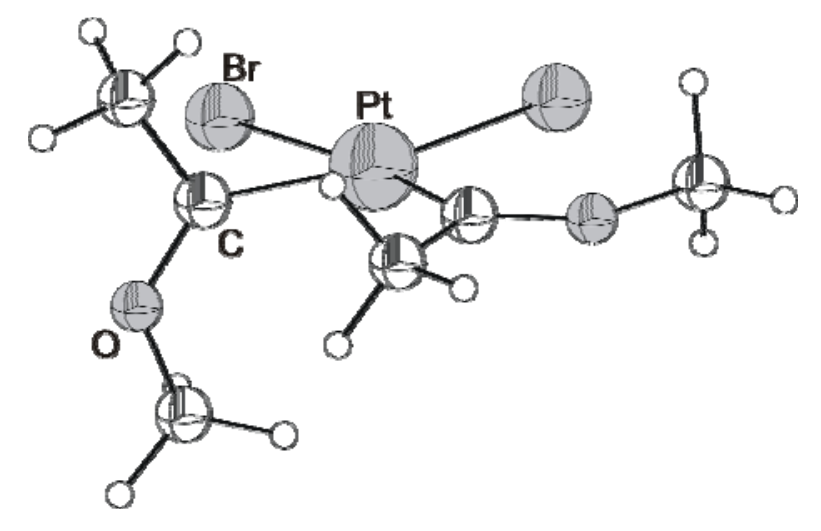

\begin{tabular}{lll}
\hline interplanar angle $^{\text {a) }}$ & $85.9^{\circ}$ & $1.2^{\circ}$ \\
$\mathrm{Pt}-\mathrm{Br}$ & $2.564 \AA$ & $2.527 \AA$ \\
$\mathrm{Pt}-\mathrm{C}$ & $1.976 \AA$ & $1.985 \AA$ \\
$\mathrm{C}-\mathrm{O}$ & $1.300 \AA$ & $1.301 \AA$
\end{tabular}

a) coordination plane $(\mathrm{C}, \mathrm{C}, \mathrm{Cl}, \mathrm{Cl})$ - ligand plane $(\mathrm{C}, \mathrm{C}, \mathrm{O}, \mathrm{C})$ 


\section{Complex 8 tc:}

trans-[PtBr 2 [C(OMe)Me $\left.)_{2}\right]$ (carbenes: $E, E$-configuration, cisoid)

file: ptbr2carbene15trans_cis.out

equilibrium structure

$\mathrm{E}(\mathrm{RB}+\mathrm{HF}-\mathrm{LYP})$

Zero-point vibrational energy

Sum of electronic and zero-point energies $\quad-5648.390258 \mathrm{Ha}$
$-5648.56592684 \mathrm{Ha}$

$110.23412 \mathrm{kcal} / \mathrm{mol}$
$\begin{array}{llll} & x & y & Z \\ \text { Pt } & 0.000023 & 0.000059 & -0.156743\end{array}$
$\begin{array}{llll}\text { C } & -0.001307 & 2.022457 & -0.242100\end{array}$
C $\quad 0.001363 \quad-2.022376 \quad-0.242100$
$\begin{array}{llll}\text { O } & -0.002043 & 2.851266 & 0.754518\end{array}$
$\begin{array}{llll}\text { C } & -0.002004 & 2.749215 & -1.546948\end{array}$
$\begin{array}{llll}\text { O } & 0.002037 & -2.851175 & 0.754518\end{array}$
$\begin{array}{llll}\text { C } & 0.001660 & -2.749127 & -1.546949\end{array}$
$\begin{array}{llll}\text { C } & -0.001856 & 2.333258 & 2.114221\end{array}$
$\begin{array}{llll}\text { C } & 0.001900 & -2.333167 & 2.114222\end{array}$
$\mathrm{H} \quad-0.002431 \quad 3.837507 \quad-1.429084$
$\mathrm{H} \quad-0.881200 \quad 2.425528 \quad-2.117454$
$\begin{array}{llll}\mathrm{H} & 0.877013 & 2.426226 & -2.118097\end{array}$
$\begin{array}{llll}\mathrm{H} & 0.002723 & -3.837419 & -1.429088\end{array}$
$\mathrm{H} \quad 0.880231 \quad-2.424934 \quad-2.118104$
$\mathrm{H} \quad-0.877983 \quad-2.426636 \quad-2.117437$
$\begin{array}{llll}\mathrm{H} & -0.002485 & 3.215765 & 2.753768\end{array}$
$\begin{array}{llll}\mathrm{H} & 0.897553 & 1.734766 & 2.264517\end{array}$
$\begin{array}{llll}\mathrm{H} & -0.900538 & 1.733627 & 2.264327\end{array}$
$\begin{array}{llll}\mathrm{H} & 0.002640 & -3.215676 & 2.753767\end{array}$
$\begin{array}{llll}\mathrm{H} & -0.897550 & -1.734750 & 2.264575\end{array}$ 


$\begin{array}{llll}\mathrm{H} & 0.900545 & -1.733466 & 2.264263 \\ \mathrm{Br} & 2.509379 & 0.001582 & -0.099655 \\ \mathrm{Br} & -2.509344 & -0.001794 & -0.099897\end{array}$

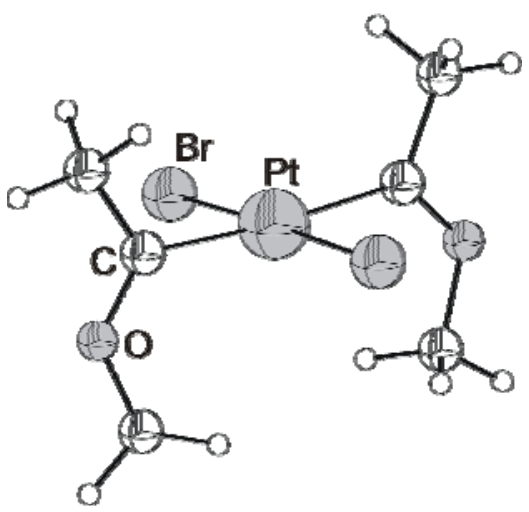

\begin{tabular}{lll}
\hline interplanar angle $^{\text {a) }}$ & $90.0^{\circ}$ & $90.0^{\circ}$ \\
$\mathrm{Pt}-\mathrm{Br}$ & $2.510 \AA$ & $2.510 \AA$ \\
$\mathrm{Pt}-\mathrm{C}$ & $2.024 \AA$ & $2.024 \AA$ \\
$\mathrm{C}-\mathrm{O}$ & $1.296 \AA$ & $1.296 \AA$
\end{tabular}

a) coordination plane $(\mathrm{C}, \mathrm{C}, \mathrm{Cl}, \mathrm{Cl})$ - ligand plane $(\mathrm{C}, \mathrm{C}, \mathrm{O}, \mathrm{C})$

\section{Complex 8 it $_{\text {: }}$}

trans-[PtBr 2 (C(OMe)Me $\left.)_{2}\right]$ (carbenes: $E, E$-configuration, transoid)

file: ptbr2carbene19trans_trans.out

equilibrium structure

$\mathrm{E}(\mathrm{RB}+\mathrm{HF}-\mathrm{LYP})$

Zero-point vibrational energies

Sum of electronic and zero-point energies $\quad-5648.391026 \mathrm{Ha}$
$-5648.56666784 \mathrm{Ha}$

$110.21719 \mathrm{kcal} / \mathrm{mol}$

$\begin{array}{llll} & x & y & Z \\ \text { Pt } & -0.029343 & -0.006935 & -0.000782 \\ \text { C } & -0.403195 & 1.914111 & -0.522331 \\ \text { C } & 0.339431 & -1.928951 & 0.520461\end{array}$




$\begin{array}{llll}\mathrm{O} & -0.446426 & 2.936439 & 0.272367 \\ \mathrm{C} & -0.668630 & 2.316433 & -1.936016 \\ \mathrm{O} & 0.740477 & -2.880869 & -0.262124 \\ \mathrm{C} & 0.173651 & -2.417080 & 1.922248 \\ \mathrm{C} & -0.219587 & 2.746394 & 1.698764 \\ \mathrm{C} & 0.954322 & -2.607771 & -1.677376 \\ \mathrm{H} & -0.848303 & 3.390422 & -2.047657 \\ \mathrm{H} & -1.532728 & 1.743263 & -2.293912 \\ \mathrm{H} & 0.188138 & 2.006621 & -2.546947 \\ \mathrm{H} & 0.434098 & -3.473673 & 2.040236 \\ \mathrm{H} & -0.869357 & -2.243860 & 2.215656 \\ \mathrm{H} & 0.791269 & -1.794630 & 2.580887 \\ \mathrm{H} & -0.364497 & 3.731478 & 2.141778 \\ \mathrm{H} & 0.799336 & 2.387017 & 1.847138 \\ \mathrm{H} & -0.944060 & 2.026827 & 2.082081 \\ \mathrm{H} & 1.214871 & -3.571501 & -2.114429 \\ \mathrm{H} & 1.768958 & -1.889691 & -1.776548 \\ \mathrm{H} & 0.033555 & -2.211662 & -2.107390 \\ \mathrm{Br} & -2.491232 & -0.475541 & -0.021646 \\ \mathrm{H} & 2.440064 & 0.471454 & 0.019464\end{array}$

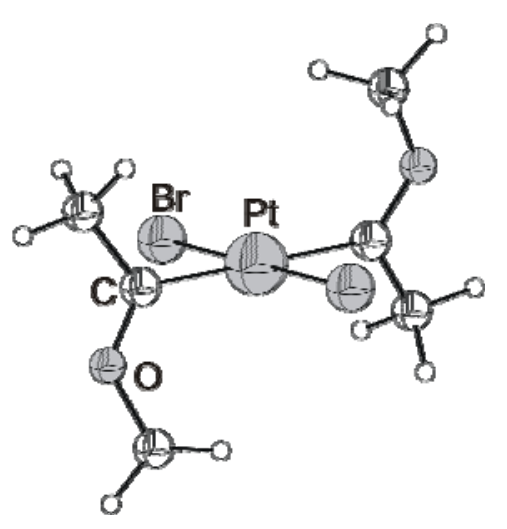

interplanar angle $^{\text {a) }} \quad 78.8^{\circ} \quad 81.3^{\circ}$ 


\begin{tabular}{lll}
\hline $\mathrm{Pt}-\mathrm{Br}$ & $2.506 \AA$ & $2.515 \AA$ \\
$\mathrm{Pt}-\mathrm{C}$ & $2.025 \AA$ & $2.025 \AA$ \\
$\mathrm{C}-\mathrm{O}$ & $1.296 \AA$ & $1.296 \AA$ \\
\hline
\end{tabular}

a) coordination plane $(\mathrm{C}, \mathrm{C}, \mathrm{Cl}, \mathrm{Cl})$ - ligand plane $(\mathrm{C}, \mathrm{C}, \mathrm{O}, \mathrm{C})$

Transition state ts $\left(\mathbf{8}_{\mathrm{tt}} \rightarrow \mathbf{8}_{\mathrm{tc}}\right)$

trans-[PtBr$\left.{ }_{2}\{\mathrm{C}(\mathrm{OMe}) \mathrm{Me})_{2}\right]$ (carbenes: $E, E$-configuration)

file: ptbr2carbene17trans_ts.out

transition structure (carbenes: transoid $\longleftrightarrow \rightarrow$ cisoid)

$\mathrm{E}(\mathrm{RB}+\mathrm{HF}-\mathrm{LYP})$

Zero-point vibrational energies

Sum of electronic and zero-point energies $\quad-5648.374913 \mathrm{Ha}$
$-5648.55109430 \mathrm{Ha}$

$110.55520 \mathrm{kcal} / \mathrm{mol}$
$\begin{array}{ccc}x & y & z\end{array}$
Pt $\quad-0.006659$
$0.110796 \quad-0.072851$
$\begin{array}{llll}\text { C } & 0.664387 & 2.060438 & 0.099579\end{array}$
$\begin{array}{llll}\text { C } & -0.805805 & -1.728477 & -0.338790\end{array}$
$\begin{array}{llll}\mathrm{O} & 1.859380 & 2.577527 & 0.156951\end{array}$
$\begin{array}{llll}\text { C } & -0.302769 & 3.211535 & 0.151364\end{array}$
$\begin{array}{llll}\mathrm{O} & -1.164858 & -2.555010 & 0.587395\end{array}$
$\begin{array}{llll}\text { C } & -1.059152 & -2.277605 & -1.704528\end{array}$
$\begin{array}{llll}\text { C } & 3.096315 & 1.826385 & 0.131285\end{array}$
$\begin{array}{llll}\text { C } & -0.994965 & -2.186813 & 1.986370\end{array}$
$\begin{array}{llll}\mathrm{H} & 0.217688 & 4.169464 & 0.241644\end{array}$
$\begin{array}{llll}\mathrm{H} & -0.996007 & 3.066596 & 0.986006\end{array}$
$\begin{array}{llll}\mathrm{H} & -0.926272 & 3.196663 & -0.748871\end{array}$
$\begin{array}{llll}\mathrm{H} & -1.493533 & -3.281739 & -1.676618\end{array}$
$\mathrm{H} \quad-0.110082 \quad-2.285626 \quad-2.252434$
$\begin{array}{llll}\mathrm{H} & -1.727807 & -1.586174 & -2.230370\end{array}$ 


$\begin{array}{llll}\mathrm{H} & 3.875850 & 2.587173 & 0.183261 \\ \mathrm{H} & 3.161558 & 1.253083 & -0.791724 \\ \mathrm{H} & 3.138515 & 1.156366 & 0.988109 \\ \mathrm{H} & -1.356828 & -3.046028 & 2.550328 \\ \mathrm{H} & -1.591350 & -1.296057 & 2.188744 \\ \mathrm{H} & 0.063926 & -2.002860 & 2.172271 \\ \mathrm{Br} & -2.440984 & 0.789324 & -0.045144 \\ \mathrm{Br} & 2.130113 & -1.251777 & -0.064411\end{array}$

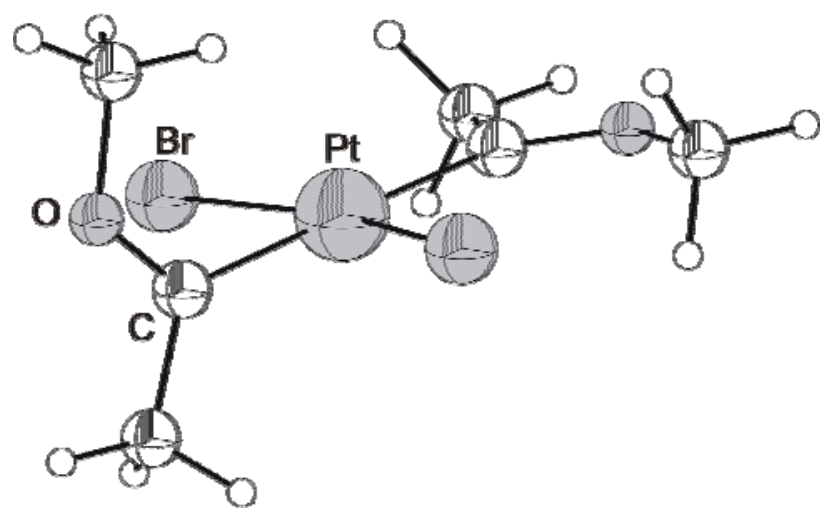

\begin{tabular}{lll}
\hline interplanar angle $^{\text {a) }}$ & $89.8^{\circ}$ & $2.2^{\circ}$ \\
$\mathrm{Pt}-\mathrm{Br}$ & $2.527 \AA$ & $2.534 \AA$ \\
$\mathrm{Pt}-\mathrm{C}$ & $2.023 \AA$ & $2.069 \AA$ \\
$\mathrm{C}-\mathrm{O}$ & $1.292 \AA$ & $1.303 \AA$
\end{tabular}

a) coordination plane $(\mathrm{C}, \mathrm{C}, \mathrm{Cl}, \mathrm{Cl})$ - ligand plane $(\mathrm{C}, \mathrm{C}, \mathrm{O}, \mathrm{C})$

\section{Complex 9c:}

cis-[PtI $\left.2 \mathrm{C}(\mathrm{OMe}) \mathrm{Me})_{2}\right]$ (carbenes: $E, E$-configuration, cisoid)

file: ptI2carbene01.out

equilibrium structure

$\mathrm{E}(\mathrm{RB}+\mathrm{HF}-\mathrm{LYP})$

Zero-point vibrational energies

Sum of electronic and zero-point energies $\quad-528.417275 \mathrm{Ha}$

$-528.592425191 \mathrm{Ha}$

$109.90844 \mathrm{kcal} / \mathrm{mol}$ 


\begin{tabular}{|c|c|c|c|}
\hline & $x$ & $y$ & $Z$ \\
\hline $\mathrm{Pt}$ & -0.000021 & 0.407606 & -0.133635 \\
\hline $\mathrm{C}$ & 1.537594 & 1.620222 & -0.382772 \\
\hline $\mathrm{C}$ & -1.537589 & 1.620253 & -0.382836 \\
\hline $\mathrm{O}$ & 2.298619 & 2.148308 & 0.530146 \\
\hline $\mathrm{C}$ & 2.000231 & 2.025505 & -1.753020 \\
\hline $\mathrm{O}$ & -2.298606 & 2.148417 & 0.530044 \\
\hline $\mathrm{C}$ & -2.000268 & 2.025394 & -1.753112 \\
\hline $\mathrm{C}$ & 2.122170 & 1.833484 & 1.933226 \\
\hline $\mathrm{C}$ & -2.122168 & 1.833684 & 1.933149 \\
\hline $\mathrm{H}$ & 2.905403 & 2.639043 & -1.713148 \\
\hline $\mathrm{H}$ & 1.200369 & 2.574964 & -2.263024 \\
\hline $\mathrm{H}$ & 2.186600 & 1.118290 & -2.337410 \\
\hline $\mathrm{H}$ & -2.904951 & 2.639651 & -1.713230 \\
\hline $\mathrm{H}$ & -2.187579 & 1.118048 & -2.337015 \\
\hline $\mathrm{H}$ & -1.200120 & 2.573922 & -2.263646 \\
\hline $\mathrm{H}$ & 2.816125 & 2.487020 & 2.460864 \\
\hline $\mathrm{H}$ & 2.367604 & 0.780039 & 2.082046 \\
\hline $\mathrm{H}$ & 1.090371 & 2.029138 & 2.228084 \\
\hline $\mathrm{H}$ & -2.816616 & 2.486783 & 2.460680 \\
\hline $\mathrm{H}$ & -1.090548 & 2.030055 & 2.228159 \\
\hline $\mathrm{H}$ & -2.366944 & 0.780086 & 2.081959 \\
\hline I & -2.018176 & -1.463956 & 0.032672 \\
\hline I & 2.018214 & -1.463880 & 0.032609 \\
\hline
\end{tabular}




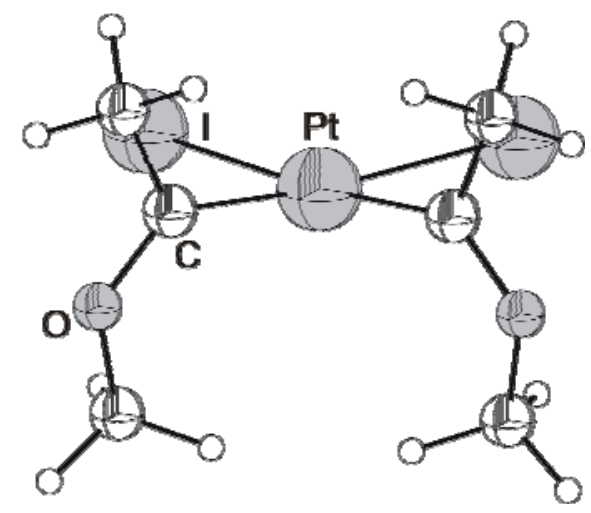

\begin{tabular}{lll}
\hline interplanar angle $^{\text {a) }}$ & $80.5^{\circ}$ & $80.6^{\circ}$ \\
$\mathrm{Pt}-\mathrm{I}$ & $2.757 \AA$ & $2.757 \AA$ \\
$\mathrm{Pt}-\mathrm{C}$ & $1.974 \AA$ & $1.974 \AA$ \\
$\mathrm{C}-\mathrm{O}$ & $1.301 \AA$ & $1.301 \AA$ \\
\hline
\end{tabular}

a) coordination plane $(\mathrm{C}, \mathrm{C}, \mathrm{Cl}, \mathrm{Cl})$ - ligand plane $(\mathrm{C}, \mathrm{C}, \mathrm{O}, \mathrm{C})$

\section{Complex 9ct:}

cis-[PtI $\left.{ }_{2}\{\mathrm{C}(\mathrm{OMe}) \mathrm{Me})_{2}\right]$ (carbenes: $E, E$-configuration, transoid)

file: $\mathrm{ptI} 2$ carbene 02. out

equilibrium structure

$\mathrm{E}(\mathrm{RB}+\mathrm{HF}-\mathrm{LYP})$

Zero-point vibrational energies

Sum of electronic and zero-point energies $\quad-528.418535 \mathrm{Ha}$
$-528.593916519 \mathrm{Ha}$

$110.05367 \mathrm{kcal} / \mathrm{mol}$

$\begin{array}{llll} & x & y & z \\ \text { Pt } & -0.000011 & 0.407052 & -0.000011 \\ \text { C } & -1.530326 & 1.639124 & 0.172329 \\ \text { C } & 1.530277 & 1.639140 & -0.172308 \\ \text { O } & -2.286033 & 2.064913 & -0.796735 \\ \text { C } & -1.969120 & 2.225846 & 1.483554 \\ \text { O } & 2.285941 & 2.064905 & 0.796803 \\ \text { C } & 1.969163 & 2.225846 & -1.483513\end{array}$




$\begin{array}{llll}\mathrm{C} & -2.098355 & 1.584928 & -2.153956 \\ \mathrm{C} & 2.098159 & 1.584855 & 2.153989 \\ \mathrm{H} & -2.967233 & 2.669693 & 1.418058 \\ \mathrm{H} & -1.248981 & 3.003210 & 1.773241 \\ \mathrm{H} & -1.946107 & 1.451301 & 2.254189 \\ \mathrm{H} & 2.967272 & 2.669690 & -1.417947 \\ \mathrm{H} & 1.249046 & 3.003217 & -1.773239 \\ \mathrm{H} & 1.946207 & 1.451301 & -2.254147 \\ \mathrm{H} & -2.722006 & 2.229559 & -2.772677 \\ \mathrm{H} & -2.431305 & 0.545863 & -2.194635 \\ \mathrm{H} & -1.045580 & 1.653906 & -2.431841 \\ \mathrm{H} & 2.721912 & 2.229334 & 2.772764 \\ \mathrm{H} & 2.430923 & 0.545730 & 2.194594 \\ \mathrm{H} & 1.045390 & 1.653992 & 2.431851 \\ \mathrm{I} & -2.025293 & -1.446217 & 0.253340 \\ \mathrm{I} & 2.025354 & -1.446120 & -0.253349\end{array}$

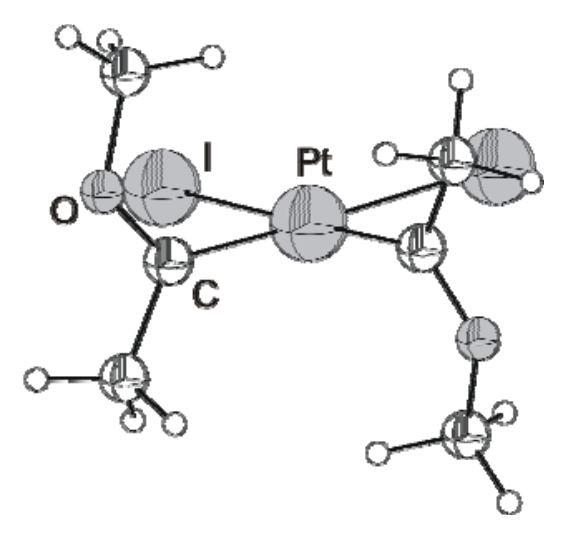

\begin{tabular}{lll}
\hline interplanar angle $^{\text {a) }}$ & $85.3^{\circ}$ & $85.3^{\circ}$ \\
$\mathrm{Pt}-\mathrm{I}$ & $2.757 \AA$ & $2.757 \AA$ \\
$\mathrm{Pt}-\mathrm{C}$ & $1.972 \AA$ & $1.972 \AA$ \\
$\mathrm{C}-\mathrm{O}$ & $1.301 \AA$ & $1.301 \AA$ \\
\hline
\end{tabular}

a) coordination plane $(\mathrm{C}, \mathrm{C}, \mathrm{Cl}, \mathrm{Cl})$ - ligand plane $(\mathrm{C}, \mathrm{C}, \mathrm{O}, \mathrm{C})$ 
Transition state ts1 $\left(9_{\mathrm{ct}} \rightarrow 9_{\mathrm{cc}}\right)$ :

cis-[PtI $\left.{ }_{2}\{\mathrm{C}(\mathrm{OMe}) \mathrm{Me})_{2}\right]$ (carbenes: $E, E$-configuration)

file: ptI2carbene05_ts.out

transition structure (carbenes: transoid $\longleftarrow \longrightarrow$ cisoid)

$\mathrm{E}(\mathrm{RB}+\mathrm{HF}-\mathrm{LYP})$

Zero-point vibrational energies

Sum of electronic and zero-point energies
$-528.571855270 \mathrm{Ha}$

$110.43389 \mathrm{kcal} / \mathrm{mol}$

$-528.395868 \mathrm{Ha}$

\begin{tabular}{|c|c|c|c|}
\hline & $x$ & $y$ & $Z$ \\
\hline $\mathrm{Pt}$ & -0.176452 & 0.377277 & -0.074493 \\
\hline $\mathrm{C}$ & -0.092745 & 2.372412 & -0.027105 \\
\hline $\mathrm{C}$ & -2.114815 & 0.036984 & -0.290999 \\
\hline $\mathrm{O}$ & -1.072486 & 3.247397 & -0.070698 \\
\hline $\mathrm{C}$ & 1.174291 & 3.172587 & 0.084986 \\
\hline $\mathrm{O}$ & -2.991348 & -0.171012 & 0.654007 \\
\hline $\mathrm{C}$ & -2.761304 & -0.137421 & -1.64215 \\
\hline $\mathrm{C}$ & -2.475721 & 2.957597 & -0.171814 \\
\hline $\mathrm{C}$ & -2.587057 & -0.213081 & 2.045768 \\
\hline $\mathrm{H}$ & 0.955659 & 4.244137 & 0.099772 \\
\hline $\mathrm{H}$ & 1.839134 & 2.922032 & -0.746891 \\
\hline $\mathrm{H}$ & 1.717136 & 2.873970 & 0.986416 \\
\hline $\mathrm{H}$ & -3.835462 & 0.074528 & -1.601510 \\
\hline $\mathrm{H}$ & -2.629258 & -1.186435 & -1.932250 \\
\hline $\mathrm{H}$ & -2.266399 & 0.473063 & -2.400479 \\
\hline $\mathrm{H}$ & -2.960337 & 3.934194 & -0.170867 \\
\hline $\mathrm{H}$ & -2.807616 & 2.375833 & 0.687317 \\
\hline $\mathrm{H}$ & -2.689208 & 2.439300 & -1.106584 \\
\hline $\mathrm{H}$ & -3.510999 & -0.127691 & 2.617502 \\
\hline $\mathrm{H}$ & -1.896130 & 0.604624 & 2.262403 \\
\hline
\end{tabular}




$$
\begin{array}{llll}
\text { H } & -2.095850 & -1.172039 & 2.224314 \\
\text { I } & -0.306705 & -2.386079 & -0.069839 \\
\text { I } & 2.563260 & 0.110067 & 0.074230
\end{array}
$$

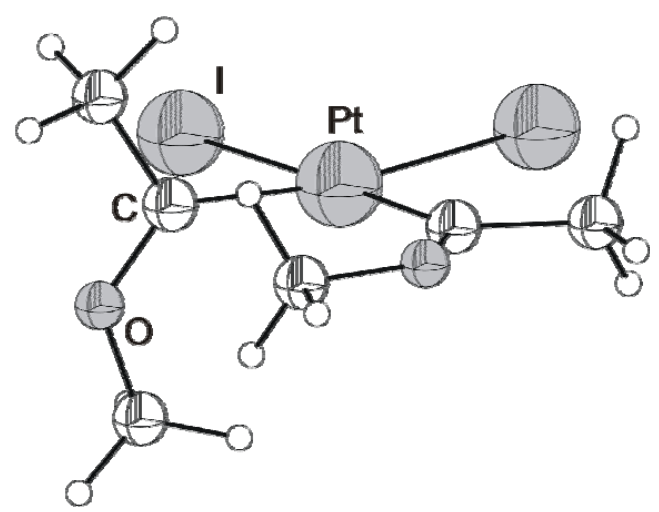

\begin{tabular}{lll}
\hline interplanar angle $^{\text {a) }}$ & $87.7^{\circ}$ & $1.4^{\circ}$ \\
$\mathrm{Pt}-\mathrm{I}$ & $2.766 \AA$ & $2.757 \AA$ \\
$\mathrm{Pt}-\mathrm{C}$ & $1.980 \AA$ & $1.997 \AA$ \\
$\mathrm{C}-\mathrm{O}$ & $1.314 \AA$ & $1.306 \AA$
\end{tabular}

a) coordination plane $(\mathrm{C}, \mathrm{C}, \mathrm{Cl}, \mathrm{Cl})$ - ligand plane $(\mathrm{C}, \mathrm{C}, \mathrm{O}, \mathrm{C})$

Transition state ts $2\left(9_{\mathrm{ct}} \rightarrow 9_{\mathrm{cc}}\right)$ :

cis-[PtI 2 $\left.\{\mathrm{C}(\mathrm{OMe}) \mathrm{Me})_{2}\right]$ (carbenes: $E, E$-configuration)

file: ptI2carbene06_ts.out

transition structure (carbenes: transoid $\longleftarrow \longrightarrow$ cisoid)

$\mathrm{E}(\mathrm{RB}+\mathrm{HF}-\mathrm{LYP}) \quad-528.570987568 \mathrm{Ha}$

Zero-point vibrational energy $\quad 110.41900 \mathrm{kcal} / \mathrm{mol}$

Sum of electronic and zero-point energies $\quad-528.395024 \mathrm{Ha}$

$\begin{array}{llcc} & x & y & z \\ \text { Pt } & 0.045236 & 0.449161 & -0.085671 \\ \text { C } & 1.153667 & 2.110800 & -0.091343 \\ \text { C } & -1.656607 & 1.454327 & -0.279235\end{array}$



$\begin{array}{lllll}\text { O } & 2.428568 & 2.356927 & -0.016908\end{array}$
$\begin{array}{llll}\text { C } & 0.477306 & 3.467343 & -0.192430\end{array}$
O $\quad-2.416159 \quad 1.889511 \quad 0.684160$
$\begin{array}{lllll}\text { C } & -2.254989 & 1.786278 & -1.618744\end{array}$
$\begin{array}{llll}\text { C } & 3.524376 & 1.414899 & 0.084127\end{array}$
$\begin{array}{llll}\text { C } & -2.121301 & 1.571377 & 2.068845\end{array}$
$\begin{array}{llll}\mathrm{H} & 1.207728 & 4.279893 & -0.153647\end{array}$
$\begin{array}{llll}\mathrm{H} & -0.245839 & 3.590462 & 0.620336\end{array}$
$\mathrm{H} \quad-0.079518 \quad 3.531490 \quad-1.133382$
$\mathrm{H} \quad-3.029884 \quad 2.555301 \quad-1.536298$
H $\quad-2.705642 \quad 0.867916 \quad-2.012085$
$\begin{array}{llll}\mathrm{H} & -1.478714 & 2.085567 & -2.327768\end{array}$
$\begin{array}{llll}\mathrm{H} & 4.411729 & 2.048132 & 0.116367\end{array}$
$\begin{array}{llll}\mathrm{H} & 3.526799 & 0.760156 & -0.784959\end{array}$
$\begin{array}{llll}\mathrm{H} & 3.418213 & 0.822841 & 0.991174\end{array}$
$\begin{array}{lllllll}\mathrm{H} & 19 & 1 & 0 & -2.713169 & 2.271832 & 2.657514\end{array}$
$\mathrm{H} \quad \begin{array}{llll}-1.051756 & 1.677239 & 2.259904\end{array}$
$\mathrm{H} \quad-2.431306 \quad 0.539062 \quad 2.244939$
I $\quad \begin{array}{llll}-1.874785 & -1.563065 & -0.071577\end{array}$
I $\quad \begin{array}{llll}1.927785 & -1.547616 & 0.082424\end{array}$

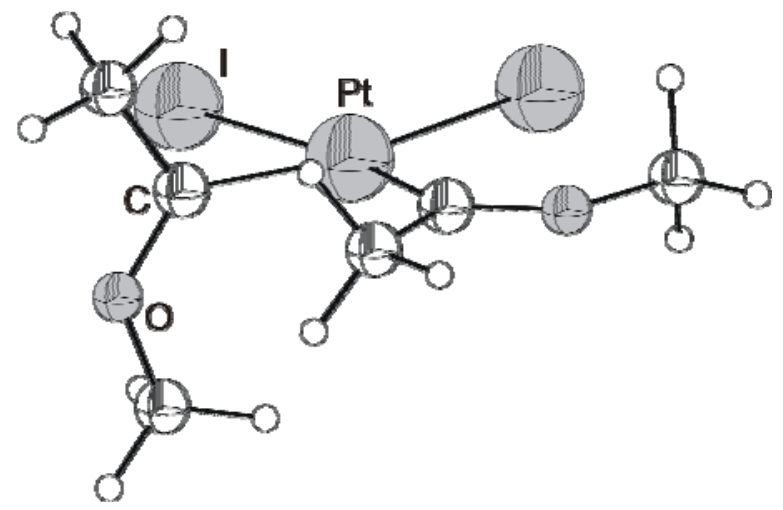

interplanar angle $^{\text {a) }} \quad 87.4^{\circ} \quad 0.8^{\circ}$ 


\begin{tabular}{lll}
\hline $\mathrm{Pt}-\mathrm{I}$ & $2.781 \AA$ & $2.749 \AA$ \\
$\mathrm{Pt}-\mathrm{C}$ & $1.986 \AA$ & $1.997 \AA$ \\
$\mathrm{C}-\mathrm{O}$ & $1.302 \AA$ & $1.301 \AA$ \\
\hline
\end{tabular}

a) coordination plane $(\mathrm{C}, \mathrm{C}, \mathrm{Cl}, \mathrm{Cl})$ - ligand plane $(\mathrm{C}, \mathrm{C}, \mathrm{O}, \mathrm{C})$

\section{Complex 9 tc:}

trans-[PtI $\left.[\mathrm{C}(\mathrm{OMe}) \mathrm{Me})_{2}\right]$ (carbenes: $E, E$-configuration, cisoid)

file: ptI2carbene07_trans_cis.out

equilibrium structure

$\mathrm{E}(\mathrm{RB}+\mathrm{HF}-\mathrm{LYP})$

Zero-point vibrational energies

Sum of electronic and zero-point energies $\quad-528.425971 \mathrm{Ha}$
$-528.601465329 \mathrm{Ha}$

$110.12459 \mathrm{kcal} / \mathrm{mol}$ $\begin{array}{lll}x & y & z\end{array}$

Pt $\quad-0.000003 \quad 0.000001 \quad-0.148286$

$\begin{array}{llll}\text { C } & 0.000133 & 2.022886 & -0.238098\end{array}$

$\begin{array}{llll}\text { C } & -0.000088 & -2.022876 & -0.238096\end{array}$

$\begin{array}{llll}\mathrm{O} & 0.000070 & 2.859031 & 0.753793\end{array}$

$\begin{array}{llll}\text { C } & 0.000178 & 2.747909 & -1.546642\end{array}$

$\begin{array}{llll}\mathrm{O} & -0.000004 & -2.859022 & 0.753795\end{array}$

$\begin{array}{llll}\text { C } & -0.000287 & -2.747900 & -1.546640\end{array}$

$\begin{array}{llll}\text { C } & -0.000081 & 2.359032 & 2.118885\end{array}$

$\begin{array}{llll}\text { C } & 0.000029 & -2.359022 & 2.118885\end{array}$

$\begin{array}{llll}\mathrm{H} & 0.001032 & 3.835964 & -1.426916\end{array}$

$\begin{array}{llll}\mathrm{H} & -0.880010 & 2.428990 & -2.117781\end{array}$

$\mathrm{H} \quad 0.879201 \quad 2.427605 \quad-2.118743$

$\begin{array}{llll}\mathrm{H} & 0.000347 & -3.835955 & -1.426912\end{array}$

$\begin{array}{llll}\mathrm{H} & 0.878778 & -2.427793 & -2.118794\end{array}$

$\begin{array}{llll}\mathrm{H} & -0.880432 & -2.428787 & -2.117728\end{array}$ 


$\begin{array}{llll}\mathrm{H} & 0.000570 & 3.249324 & 2.747283 \\ \mathrm{H} & 0.898917 & 1.761638 & 2.278035 \\ \mathrm{H} & -0.899824 & 1.762807 & 2.278212 \\ \mathrm{H} & 0.000408 & -3.249314 & 2.747284 \\ \mathrm{H} & -0.899521 & -1.762511 & 2.278231 \\ \mathrm{H} & 0.899221 & -1.761914 & 2.278016 \\ \mathrm{I} & 2.705607 & -0.000052 & -0.073443 \\ \mathrm{I} & -2.705575 & 0.000044 & -0.073431\end{array}$

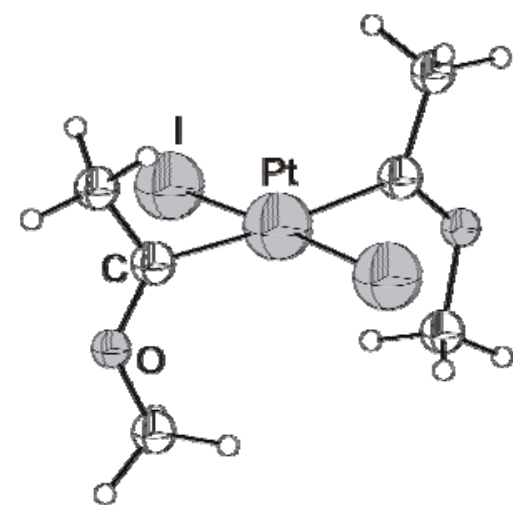

\begin{tabular}{lll}
\hline interplanar angle $^{\text {a) }}$ & $90.0^{\circ}$ & $90.0^{\circ}$ \\
$\mathrm{Pt}-\mathrm{I}$ & $2.707 \AA$ & $2.707 \AA$ \\
$\mathrm{Pt}-\mathrm{C}$ & $2.025 \AA$ & $2.025 \AA$ \\
$\mathrm{C}-\mathrm{O}$ & $1.297 \AA$ & $1.297 \AA$ \\
\hline
\end{tabular}

a) coordination plane $(\mathrm{C}, \mathrm{C}, \mathrm{Cl}, \mathrm{Cl})$ - ligand plane $(\mathrm{C}, \mathrm{C}, \mathrm{O}, \mathrm{C})$

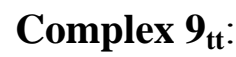

trans-[PtI 2 $\left.\{\mathrm{C}(\mathrm{OMe}) \mathrm{Me})_{2}\right]$ (carbenes: $E, E$-configuration, transoid)

file: ptI2carbene08_trans_trans.out

equilibrium structure

$\mathrm{E}(\mathrm{RB}+\mathrm{HF}-\mathrm{LYP})$

$-528.601829525 \mathrm{Ha}$

Zero-point vibrational energies

$109.81082 \mathrm{kcal} / \mathrm{mol}$ 
Sum of electronic and zero-point energies $\quad-528.426835 \mathrm{Ha}$

\begin{tabular}{|c|c|c|c|}
\hline & $x$ & $y$ & $Z$ \\
\hline $\mathrm{Pt}$ & -0.000629 & 0.000014 & 0.000009 \\
\hline $\mathrm{C}$ & -0.000537 & -1.954078 & 0.534373 \\
\hline $\mathrm{C}$ & -0.000434 & 1.954099 & -0.534356 \\
\hline $\mathrm{O}$ & 0.003310 & -2.974447 & -0.266539 \\
\hline $\mathrm{C}$ & -0.005058 & -2.396236 & 1.963471 \\
\hline $\mathrm{O}$ & 0.003361 & 2.974489 & 0.266534 \\
\hline $\mathrm{C}$ & -0.004803 & 2.396238 & -1.963457 \\
\hline $\mathrm{C}$ & 0.007847 & -2.759715 & -1.705651 \\
\hline $\mathrm{C}$ & 0.007861 & 2.759788 & 1.705655 \\
\hline $\mathrm{H}$ & -0.002107 & -3.485720 & 2.069078 \\
\hline $\mathrm{H}$ & -0.888761 & -1.970095 & 2.453526 \\
\hline $\mathrm{H}$ & 0.871364 & -1.964367 & 2.461423 \\
\hline $\mathrm{H}$ & -0.002134 & 3.485722 & -2.069050 \\
\hline $\mathrm{H}$ & -0.888252 & 1.969835 & -2.453733 \\
\hline $\mathrm{H}$ & 0.871877 & 1.964634 & -2.461191 \\
\hline $\mathrm{H}$ & 0.008480 & -3.758579 & -2.141222 \\
\hline $\mathrm{H}$ & 0.907845 & -2.206689 & -1.978534 \\
\hline $\mathrm{H}$ & -0.889585 & -2.205378 & -1.984247 \\
\hline $\mathrm{H}$ & 0.008202 & 3.758664 & 2.141197 \\
\hline $\mathrm{H}$ & 0.908003 & 2.207024 & 1.978597 \\
\hline $\mathrm{H}$ & -0.889427 & 2.205200 & 1.984205 \\
\hline I & 2.706353 & -0.000052 & 0.000009 \\
\hline I & -2.707278 & 0.000009 & -0.000026 \\
\hline
\end{tabular}




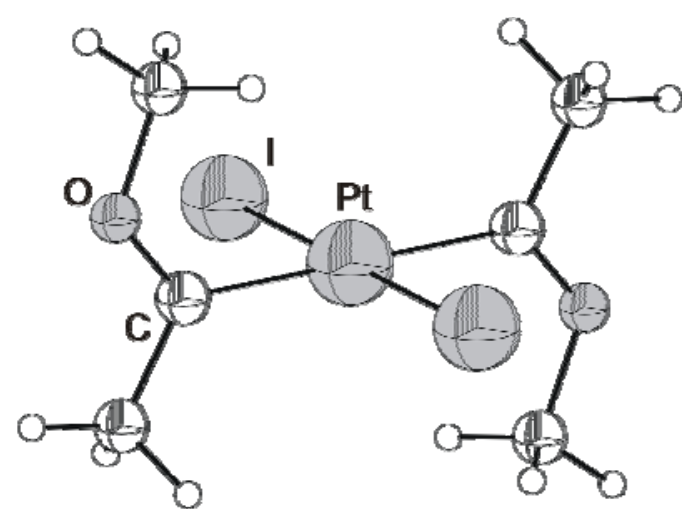

\begin{tabular}{lll}
\hline interplanar angle $^{\text {a) }}$ & $89.8^{\circ}$ & $89.9^{\circ}$ \\
$\mathrm{Pt}-\mathrm{I}$ & $2.707 \AA$ & $2.707 \AA$ \\
$\mathrm{Pt}-\mathrm{C}$ & $2.026 \AA$ & $2.026 \AA$ \\
$\mathrm{C}-\mathrm{O}$ & $1.297 \AA$ & $1.297 \AA$ \\
\hline
\end{tabular}

a) coordination plane $(\mathrm{C}, \mathrm{C}, \mathrm{Cl}, \mathrm{Cl})$ - ligand plane $(\mathrm{C}, \mathrm{C}, \mathrm{O}, \mathrm{C})$

Transition state $\operatorname{ts}\left(9_{\mathrm{tt}} \rightarrow \mathbf{9}_{\mathrm{tc}}\right)$ :

trans-[PtI 2 (C(OMe)Me $\left.)_{2}\right]$ (carbenes: E,E-configuration)

file: ptI2carbene10trans_ts.out

transition structure (carbenes: transoid $\longleftarrow \longrightarrow$ cisoid)

$\mathrm{E}(\mathrm{RB}+\mathrm{HF}-\mathrm{LYP}) \quad-528.579194903 \mathrm{Ha}$

Zero-point vibrational energies $\quad 110.26951 \mathrm{kcal} / \mathrm{mol}$

Sum of electronic and zero-point Energies $\quad-528.403469 \mathrm{Ha}$

$\begin{array}{llll} & x & y & z \\ \text { Pt } & -0.027845 & 0.188669 & -0.062589 \\ \text { C } & -0.049381 & 2.259950 & 0.102250 \\ \text { C } & -0.163479 & -1.816822 & -0.321340 \\ \text { O } & 0.899199 & 3.153097 & 0.161968 \\ \text { C } & -1.342403 & 3.030428 & 0.147706 \\ \text { O } & -0.234508 & -2.719060 & 0.604534 \\ \text { C } & -0.218308 & -2.430404 & -1.687250\end{array}$




$\begin{array}{llll}\mathrm{C} & 2.318320 & 2.881952 & 0.147665 \\ \mathrm{C} & -0.214563 & -2.334918 & 2.006005 \\ \mathrm{H} & -1.164175 & 4.106321 & 0.233819 \\ \mathrm{H} & -1.951173 & 2.674442 & 0.985150 \\ \mathrm{H} & -1.926390 & 2.812611 & -0.752758 \\ \mathrm{H} & -0.232765 & -3.523774 & -1.644725 \\ \mathrm{H} & 0.640597 & -2.082868 & -2.269812 \\ \mathrm{H} & -1.121025 & -2.062855 & -2.188763 \\ \mathrm{H} & 2.784355 & 3.866498 & 0.200303 \\ \mathrm{H} & 2.588517 & 2.368012 & -0.773366 \\ \mathrm{H} & 2.587212 & 2.274448 & 1.010273 \\ \mathrm{H} & -0.287811 & -3.272011 & 2.556893 \\ \mathrm{H} & -1.070148 & -1.687985 & 2.206617 \\ \mathrm{H} & 0.725577 & -1.822572 & 2.216607 \\ \mathrm{I} & -2.757564 & -0.075713 & -0.045332 \\ \mathrm{I} & 2.631158 & -0.516360 & -0.056564\end{array}$

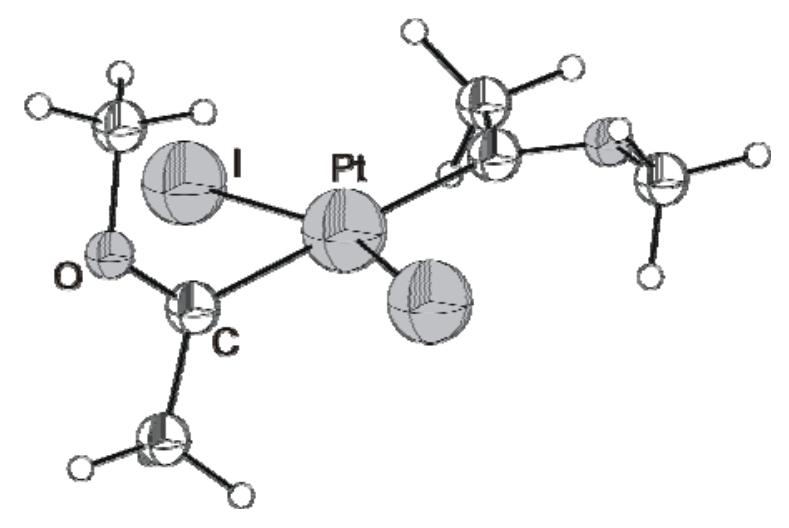

\begin{tabular}{lll}
\hline interplanar angle $^{\text {a) }}$ & $89.8^{\circ}$ & $1.9^{\circ}$ \\
$\mathrm{Pt}-\mathrm{I}$ & $2.743 \AA$ & $2.751 \AA$ \\
$\mathrm{Pt}-\mathrm{C}$ & $2.027 \AA$ & $2.078 \AA$ \\
$\mathrm{C}-\mathrm{O}$ & $1.295 \AA$ & $1.304 \AA$
\end{tabular}

a) coordination plane $(\mathrm{C}, \mathrm{C}, \mathrm{Cl}, \mathrm{Cl})$ - ligand plane $(\mathrm{C}, \mathrm{C}, \mathrm{O}, \mathrm{C})$ 


\section{Complex 10EZ1:}

cis-[PtCl$\left.{ }_{2}\{\mathrm{C}(\mathrm{OMe}) \mathrm{Me})_{2}\right]$ (carbenes: E,Z-configuration)

file: ptc12carbene10.out

equilibrium structure

$\mathrm{E}(\mathrm{RB}+\mathrm{HF}-\mathrm{LYP})$

Zero-point vibrational energies
$-1426.11817512 \mathrm{Ha}$

$111.04174 \mathrm{kcal} / \mathrm{mol}$

Sum of electronic and zero-point energies $\quad-1425.941219 \mathrm{Ha}$

\begin{tabular}{llll} 
& \multicolumn{1}{l}{ X } & \multicolumn{1}{l}{ Z } \\
$\mathrm{Pt}$ & -0.186296 & -0.290798 & -0.067530 \\
$\mathrm{Cl}$ & -2.573527 & -0.570096 & -0.321251 \\
$\mathrm{C}$ & 1.725850 & -0.018156 & 0.166347 \\
$\mathrm{C}$ & -0.650748 & 1.607774 & -0.260559 \\
$\mathrm{Cl}$ & 0.135555 & -2.672322 & -0.038130 \\
$\mathrm{O}$ & 2.278203 & 1.047003 & -0.385842 \\
$\mathrm{C}$ & 2.637109 & -0.919650 & 0.942860 \\
$\mathrm{O}$ & -1.149982 & 2.378229 & 0.655256 \\
$\mathrm{C}$ & -0.578609 & 2.306105 & -1.581225 \\
$\mathrm{C}$ & 3.697820 & 1.329985 & -0.311107 \\
$\mathrm{C}$ & -1.428888 & 1.859989 & 1.986496 \\
$\mathrm{H}$ & 3.391639 & -1.361281 & 0.276558 \\
$\mathrm{H}$ & 2.072783 & -1.730182 & 1.396906 \\
$\mathrm{H}$ & 3.171674 & -0.345055 & 1.712455 \\
$\mathrm{H}$ & -0.813434 & 3.372067 & -1.501051 \\
$\mathrm{H}$ & -1.310374 & 1.816122 & -2.237196 \\
$\mathrm{H}$ & 0.406271 & 2.152721 & -2.030576 \\
$\mathrm{H}$ & 3.834227 & 2.236377 & -0.899374 \\
$\mathrm{H}$ & 4.274690 & 0.509620 & -0.743556 \\
$\mathrm{H}$ & 4.001368 & 1.504253 & 0.724170
\end{tabular}




$\begin{array}{llll}\mathrm{H} & -1.702768 & 2.733584 & 2.577304 \\ \mathrm{H} & -0.538709 & 1.368667 & 2.382249 \\ \mathrm{H} & -2.251758 & 1.148298 & 1.906724\end{array}$

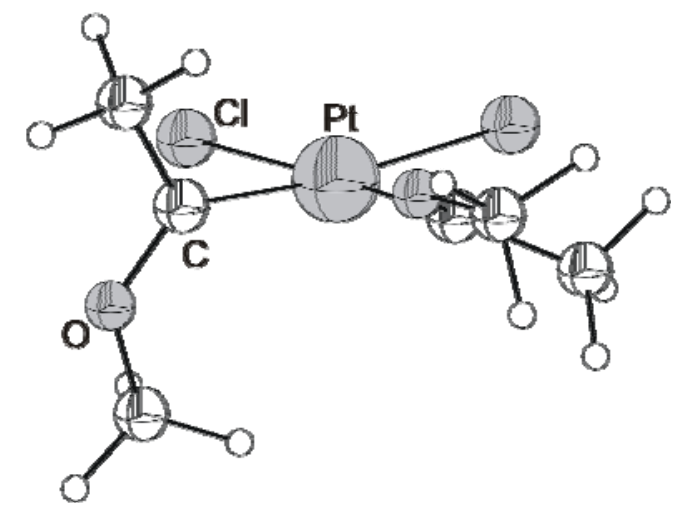

\begin{tabular}{lll}
\hline interplanar angle $^{\text {a) }}$ & $81.0^{\circ}$ & $31.0^{\circ}$ \\
$\mathrm{Pt}-\mathrm{Cl}$ & $2.417 \AA$ & $2.403 \AA$ \\
$\mathrm{Pt}-\mathrm{C}$ & $1.964 \AA$ & $1.946 \AA$ \\
$\mathrm{C}-\mathrm{O}$ & $1.297 \AA$ & $1.321 \AA$
\end{tabular}

a) coordination plane $(\mathrm{C}, \mathrm{C}, \mathrm{Cl}, \mathrm{Cl})$ - ligand plane $(\mathrm{C}, \mathrm{C}, \mathrm{O}, \mathrm{C})$

\section{Complex 10}

cis-[PtCl 2 $\left.\{\mathrm{C}(\mathrm{OMe}) \mathrm{Me})_{2}\right]$ (carbenes: $E, Z$-configuration)

file: ptc12carbene34.out

equilibrium structure

$\mathrm{E}(\mathrm{RB}+\mathrm{HF}-\mathrm{LYP})$

Zero-point vibrational energies

Sum of electronic and zero-point energies $\quad-1425.941291 \mathrm{Ha}$
$-1426.11809756 \mathrm{Ha}$

$110.94810 \mathrm{kcal} / \mathrm{mol}$

$\begin{array}{llll} & x & y & Z \\ \text { Pt } & -0.227110 & -0.285376 & -0.069775 \\ \text { Cl } & -2.632553 & -0.198679 & -0.256855 \\ \text { C } & 1.715987 & -0.262599 & 0.088973\end{array}$




$\begin{array}{llll}\mathrm{C} & -0.387510 & 1.658676 & -0.303430 \\ \mathrm{Cl} & -0.321457 & -2.679765 & 0.069311 \\ \mathrm{O} & 2.334330 & 0.874267 & -0.196591 \\ \mathrm{C} & 2.589986 & -1.397185 & 0.525675 \\ \mathrm{O} & -0.730287 & 2.516332 & 0.606357 \\ \mathrm{C} & -0.252758 & 2.311559 & -1.642776 \\ \mathrm{C} & 3.772550 & 1.032879 & -0.130641 \\ \mathrm{C} & -1.044464 & 2.076676 & 1.958277 \\ \mathrm{H} & 3.350152 & -1.614289 & -0.237581 \\ \mathrm{H} & 1.990105 & -2.288231 & 0.699098 \\ \mathrm{H} & 3.121620 & -1.120488 & 1.447908 \\ \mathrm{H} & -0.284956 & 3.403619 & -1.577599 \\ \mathrm{H} & -1.095111 & 1.955754 & -2.251116 \\ \mathrm{H} & 0.662464 & 1.973677 & -2.135490 \\ \mathrm{H} & 3.951853 & 2.073576 & -0.397668 \\ \mathrm{H} & 4.263694 & 0.370882 & -0.847707 \\ \mathrm{H} & 4.138034 & 0.834705 & 0.879683 \\ \mathrm{H} & -1.169962 & 2.995332 & 2.530598 \\ \mathrm{H} & -0.224951 & 1.470062 & 2.346539 \\ \mathrm{H} & -1.965302 & 1.493440 & 1.919395\end{array}$

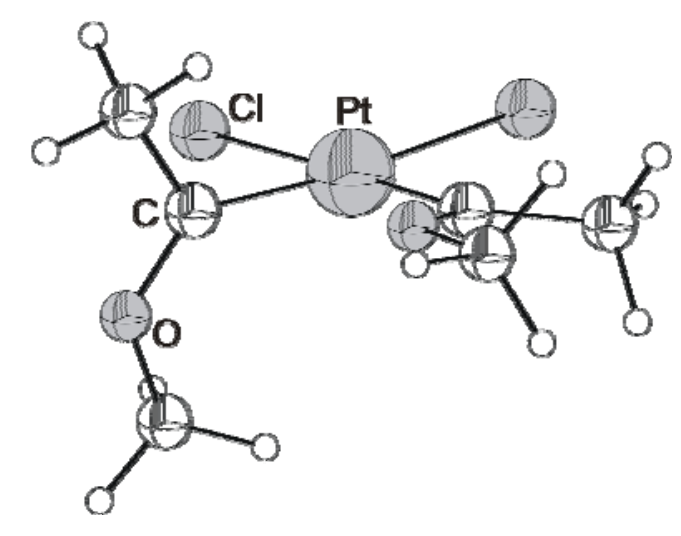

\begin{tabular}{lll}
\hline interplanar angle $^{\text {a) }}$ & $80.9^{\circ}$ & $12.5^{\circ}$
\end{tabular} 


\begin{tabular}{lll}
\hline $\mathrm{Pt}-\mathrm{Cl}$ & $2.414 \AA$ & $2.400 \AA$ \\
$\mathrm{Pt}-\mathrm{C}$ & $1.965 \AA$ & $1.950 \AA$ \\
$\mathrm{C}-\mathrm{O}$ & $1.296 \AA$ & $1.325 \AA$ \\
\hline
\end{tabular}

a) coordination plane $(\mathrm{C}, \mathrm{C}, \mathrm{Cl}, \mathrm{Cl})$ - ligand plane $(\mathrm{C}, \mathrm{C}, \mathrm{O}, \mathrm{C})$

\section{Complex 10 EZ3:}

cis-[ $\left.\mathrm{PtCl}_{2}\{\mathrm{C}(\mathrm{OMe}) \mathrm{Me})_{2}\right]$ (carbenes: E,Z-configuration)

file: ptcl2carbene09.out

equilibrium structure

$\mathrm{E}(\mathrm{RB}+\mathrm{HF}-\mathrm{LYP})$

Zero-point vibrational energies

Sum of electronic and zero-point energies $\quad-1425.941506 \mathrm{Ha}$
$-1426.11818973 \mathrm{Ha}$

$110.87077 \mathrm{kcal} / \mathrm{mol}$

$\begin{array}{lll}x & y & z\end{array}$

$\begin{array}{llll}\mathrm{Pt} & -0.231507 & -0.286938 & -0.068935\end{array}$

Cl $\quad-2.635696 \quad-0.205863 \quad-0.268351$

$\begin{array}{llll}\text { C } & 1.715619 & -0.276532 & -0.007241\end{array}$

$\begin{array}{llll}\text { C } & -0.371714 & 1.648419 & -0.382894\end{array}$

$\begin{array}{llll}\mathrm{Cl} & -0.331944 & -2.650337 & 0.335774\end{array}$

$\begin{array}{llll}\text { O } & 2.313787 & 0.889383 & 0.190854\end{array}$

$\begin{array}{llll}\text { C } & 2.612380 & -1.457322 & -0.214765\end{array}$

$\begin{array}{llll}\text { O } & -0.726590 & 2.533118 & 0.494349\end{array}$

$\begin{array}{llll}\text { C } & -0.186112 & 2.266796 & -1.732880\end{array}$

$\begin{array}{llll}\text { C } & 3.753885 & 1.041163 & 0.239105\end{array}$

$\begin{array}{llll}\text { C } & -1.053475 & 2.136755 & 1.857792\end{array}$

$\begin{array}{llll}\mathrm{H} & 3.183773 & -1.318808 & -1.144968\end{array}$

$\begin{array}{llll}\mathrm{H} & 2.021334 & -2.368485 & -0.282539\end{array}$

$\mathrm{H} \quad 3.337783 \quad-1.552725 \quad 0.604466$

$\begin{array}{llll}\mathrm{H} & -0.254869 & 3.358610 & -1.705263\end{array}$ 


$\begin{array}{llll}\mathrm{H} & -0.974620 & 1.861823 & -2.380504 \\ \mathrm{H} & 0.768679 & 1.947521 & -2.161458 \\ \mathrm{H} & 3.918832 & 2.110972 & 0.360682 \\ \mathrm{H} & 4.212389 & 0.691234 & -0.688700 \\ \mathrm{H} & 4.165767 & 0.498764 & 1.093403 \\ \mathrm{H} & -1.193458 & 3.074180 & 2.395225 \\ \mathrm{H} & -0.230773 & 1.553672 & 2.274046 \\ \mathrm{H} & -1.968444 & 1.544152 & 1.830026\end{array}$

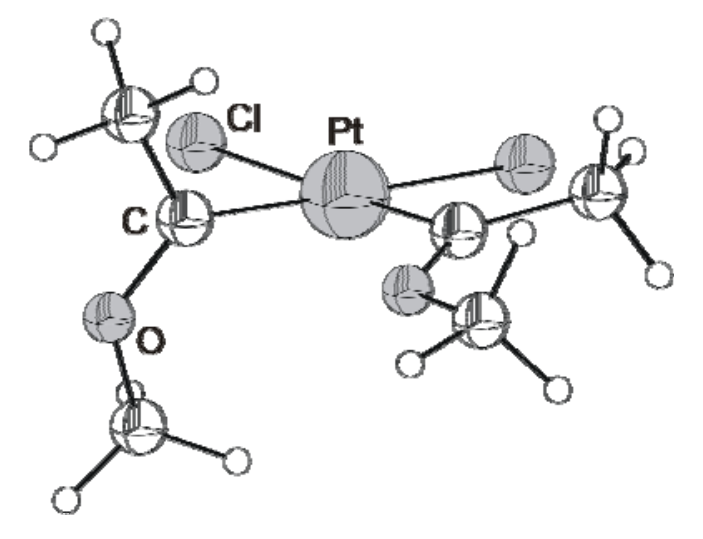

\begin{tabular}{lll}
\hline interplanar angle $^{\text {a) }}$ & $78.2^{\circ}$ & $20.0^{\circ}$ \\
$\mathrm{Pt}-\mathrm{Cl}$ & $2.414 \AA$ & $2.400 \AA$ \\
$\mathrm{Pt}-\mathrm{C}$ & $1.966 \AA$ & $1.948 \AA$ \\
$\mathrm{C}-\mathrm{O}$ & $1.295 \AA$ & $1.325 \AA$
\end{tabular}

a) coordination plane $(\mathrm{C}, \mathrm{C}, \mathrm{Cl}, \mathrm{Cl})$ - ligand plane $(\mathrm{C}, \mathrm{C}, \mathrm{O}, \mathrm{C})$

Transition state ts1 $\left(7_{\mathrm{cc}} \rightarrow 10_{\mathrm{EZ1}}\right)$ :

cis-[PtCl 2 (C(OMe)Me $\left.)_{2}\right]$

file: ptcl2carbene21_OMe_ts.out

transition structure (carbenes: E,E-configuration $\longleftrightarrow \longrightarrow E, Z$-configuration)

$\mathrm{E}(\mathrm{RB}+\mathrm{HF}-\mathrm{LYP}) \quad-1426.09811981 \mathrm{Ha}$

Zero-point vibrational energies $\quad 110.17291 \mathrm{kcal} / \mathrm{mol}$ 
Sum of electronic and zero-point energies $\quad-1425.922548 \mathrm{Ha}$

\begin{tabular}{llll} 
& \multicolumn{1}{l}{ X } & \multicolumn{1}{l}{ Z } \\
$\mathrm{Pt}$ & -0.052547 & -0.260253 & -0.014377 \\
$\mathrm{Cl}$ & -2.065270 & -1.365765 & -0.799065 \\
$\mathrm{C}$ & 1.559259 & 0.606591 & 0.550616 \\
$\mathrm{C}$ & -1.182205 & 1.352933 & -0.179831 \\
$\mathrm{Cl}$ & 1.191838 & -2.319988 & -0.046846 \\
$\mathrm{O}$ & 2.027154 & 1.704004 & -0.075044 \\
$\mathrm{C}$ & 2.399437 & 0.203997 & 1.717347 \\
$\mathrm{O}$ & -2.134641 & 1.710561 & 0.618981 \\
$\mathrm{C}$ & -1.068493 & 2.274007 & -1.350584 \\
$\mathrm{C}$ & 2.959133 & 1.508006 & -1.164873 \\
$\mathrm{C}$ & -2.472080 & 0.912409 & 1.790081 \\
$\mathrm{H}$ & 2.211814 & 0.948551 & 2.507733 \\
$\mathrm{H}$ & 3.468717 & 0.264916 & 1.476455 \\
$\mathrm{H}$ & 2.140631 & -0.789010 & 2.080958 \\
$\mathrm{H}$ & -1.769440 & 3.111920 & -1.290459 \\
$\mathrm{H}$ & -1.263836 & 1.684758 & -2.255319 \\
$\mathrm{H}$ & -0.036812 & 2.634856 & -1.418693 \\
$\mathrm{H}$ & 3.212011 & 2.507792 & -1.515537 \\
$\mathrm{H}$ & 2.480987 & 0.924984 & -1.957568 \\
$\mathrm{H}$ & 3.859265 & 0.993422 & -0.813516 \\
$\mathrm{H}$ & -3.147079 & 1.538930 & 2.371979 \\
$\mathrm{H}$ & -1.562712 & 0.673449 & 2.343629 \\
$\mathrm{H}$ & -0.001235 & 1.444181
\end{tabular}




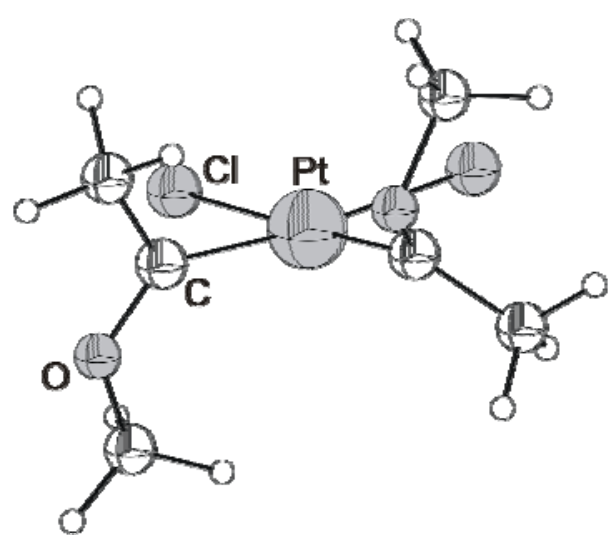

\begin{tabular}{lll}
\hline interplanar angle $^{\text {a) }}$ & $75.9^{\circ}$ & $72.5^{\circ}$ \\
$\mathrm{Pt}-\mathrm{Cl}$ & $2.427 \AA$ & $2.407 \AA$ \\
$\mathrm{Pt}-\mathrm{C}$ & $1.976 \AA$ & $1.915 \AA$ \\
$\mathrm{C}-\mathrm{O}$ & $1.293 \AA$ & $1.347 \AA$ \\
\hline
\end{tabular}

a) coordination plane $(\mathrm{C}, \mathrm{C}, \mathrm{Cl}, \mathrm{Cl})$ - ligand plane $(\mathrm{C}, \mathrm{C}, \mathrm{O}, \mathrm{C})$

Transition state ts2 $\left(7_{\mathrm{cc}} \rightarrow 10_{\mathrm{EZ1}}\right)$ :

cis- $\left[\mathrm{PtCl}_{2}\{\mathrm{C}(\mathrm{OMe}) \mathrm{Me})_{2}\right]$

file: ptcl2carbene22_OMe_ts.out

transition structure (carbenes: E,E-configuration $\longleftrightarrow \longrightarrow E, Z$-configuration)

$\mathrm{E}(\mathrm{RB}+\mathrm{HF}-\mathrm{LYP})$

Zero-point vibrational energies

Sum of electronic and zero-point energies $\quad-1425.922190 \mathrm{Ha}$
$-1426.09772709 \mathrm{Ha}$

$110.15117 \mathrm{kcal} / \mathrm{mol}$
$x$
$y$
Z
Pt $\quad \begin{array}{llll}0.072911 & -0.333723 & 0.074909\end{array}$
$\mathrm{Cl} \quad 2.380660 \quad-1.076886 \quad 0.154861$
$\begin{array}{llll}\text { C } & -1.730688 & 0.286996 & 0.280203\end{array}$
C $\quad 0.880336 \quad 1.422025 \quad 0.499310$
$\mathrm{Cl} \quad-0.657493 \quad-2.523422 \quad-0.552576$
$\begin{array}{llll}\text { O } & -2.063500 & 1.589982 & 0.130716\end{array}$ 

$\begin{array}{llll}\text { C } & -2.858853 & -0.543702 & 0.790462\end{array}$
O $\quad 1.543760 \quad 2.164574 \quad-0.327338$
$\begin{array}{llll}\text { C } & 0.873323 & 2.011455 & 1.873751\end{array}$
$\begin{array}{llll}\text { C } & -2.529615 & 2.016924 & -1.163398\end{array}$
C $\quad 1.765101 \quad 1.755365 \quad-1.707822$
$\begin{array}{llll}\mathrm{H} & -3.826904 & -0.051067 & 0.652694\end{array}$
$\mathrm{H} \quad \begin{array}{llll}-2.695723 & -0.698749 & 1.868318\end{array}$
$\begin{array}{llll}\mathrm{H} & -2.821055 & -1.541622 & 0.340214\end{array}$
$\begin{array}{llll}\mathrm{H} & 1.389999 & 2.974798 & 1.916496\end{array}$
$\begin{array}{llll}\mathrm{H} & 1.363599 & 1.292650 & 2.542197\end{array}$
H $\quad-0.160204 \quad 2.115568 \quad 2.220212$
$\begin{array}{llll}\mathrm{H} & -2.709821 & 3.087832 & -1.075254\end{array}$
H $\quad \begin{array}{llll}-3.459807 & 1.501714 & -1.427225\end{array}$
H $\quad \begin{array}{llll}-1.767496 & 1.817558 & -1.924428\end{array}$
$\mathrm{H} \quad 2.111137 \quad 2.655341 \quad-2.215109$
H $\quad 0.834002 \quad 1.380441 \quad-2.135110$
$\begin{array}{llll}\mathrm{H} & 2.521650 & 0.970362 & -1.706820\end{array}$

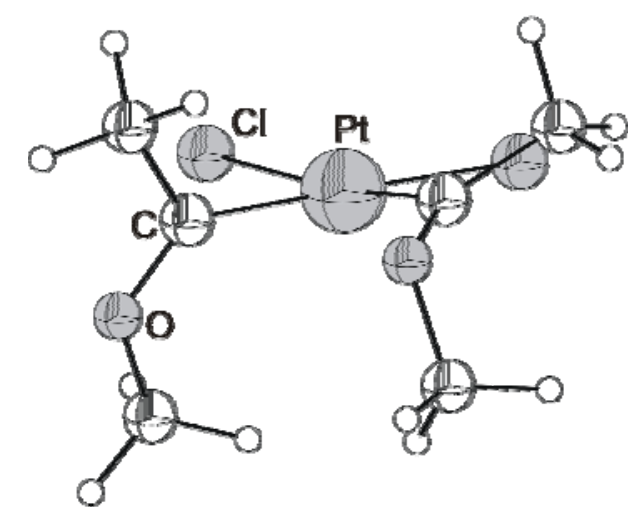

\begin{tabular}{lll}
\hline interplanar angle $^{\text {a) }}$ & $72.0^{\circ}$ & $52.5^{\circ}$ \\
$\mathrm{Pt}-\mathrm{Cl}$ & $2.392 \AA$ & $2.426 \AA$ \\
$\mathrm{Pt}-\mathrm{C}$ & $1.979 \AA$ & $1.918 \AA$ \\
$\mathrm{C}-\mathrm{O}$ & $1.294 \AA$ & $1.353 \AA$
\end{tabular}

a) coordination plane $(\mathrm{C}, \mathrm{C}, \mathrm{Cl}, \mathrm{Cl})$ - ligand plane $(\mathrm{C}, \mathrm{C}, \mathrm{O}, \mathrm{C})$ 


\section{Complex 11 zz1 $_{\text {: }}$}

cis-[PtCl 2 $\left.\{\mathrm{C}(\mathrm{OMe}) \mathrm{Me})_{2}\right]$ (carbenes: Z,Z-configuration)

file: ptc12carbene11.out

equilibrium structure

$\mathrm{E}(\mathrm{RB}+\mathrm{HF}-\mathrm{LYP})$

Zero-point vibrational energies
$-1426.10741227 \mathrm{Ha}$

$110.89937 \mathrm{kcal} / \mathrm{mol}$

Sum of electronic and zero-point energies $\quad-1425.930683 \mathrm{Ha}$

\begin{tabular}{|c|c|c|c|}
\hline & $x$ & $y$ & $Z$ \\
\hline $\mathrm{Pt}$ & 0.460524 & -0.005944 & -0.010253 \\
\hline $\mathrm{Cl}$ & 2.280237 & 1.559090 & -0.085527 \\
\hline $\mathrm{C}$ & -0.935878 & -1.358216 & -0.087331 \\
\hline $\mathrm{C}$ & -0.804806 & 1.478380 & 0.070283 \\
\hline $\mathrm{Cl}$ & 2.031937 & -1.831275 & 0.107968 \\
\hline $\mathrm{O}$ & -1.973689 & -1.263049 & 0.713456 \\
\hline $\mathrm{C}$ & -0.899307 & -2.524898 & -1.028409 \\
\hline $\mathrm{O}$ & -1.881956 & 1.438736 & -0.680533 \\
\hline $\mathrm{C}$ & -0.587746 & 2.639526 & 0.984445 \\
\hline $\mathrm{C}$ & -3.030638 & -2.254890 & 0.751061 \\
\hline $\mathrm{H}$ & -0.845646 & -3.469141 & -0.470029 \\
\hline $\mathrm{H}$ & -0.024895 & -2.463684 & -1.671501 \\
\hline $\mathrm{H}$ & -1.814815 & -2.545029 & -1.636732 \\
\hline $\mathrm{H}$ & -1.333651 & 3.435312 & 0.934154 \\
\hline $\mathrm{H}$ & 0.416163 & 3.029460 & 0.768487 \\
\hline $\mathrm{H}$ & -0.528446 & 2.253192 & 2.009565 \\
\hline $\mathrm{H}$ & -3.697566 & -1.924725 & 1.546212 \\
\hline $\mathrm{H}$ & -2.621207 & -3.239102 & 0.988145 \\
\hline $\mathrm{H}$ & -3.563780 & -2.282957 & -0.202549 \\
\hline
\end{tabular}




$\begin{array}{llll}\mathrm{C} & -2.894631 & 2.478369 & -0.687627 \\ \mathrm{H} & -2.467014 & 3.424942 & -1.024227 \\ \mathrm{H} & -3.639301 & 2.132100 & -1.403276 \\ \mathrm{H} & -3.344440 & 2.585295 & 0.302054\end{array}$

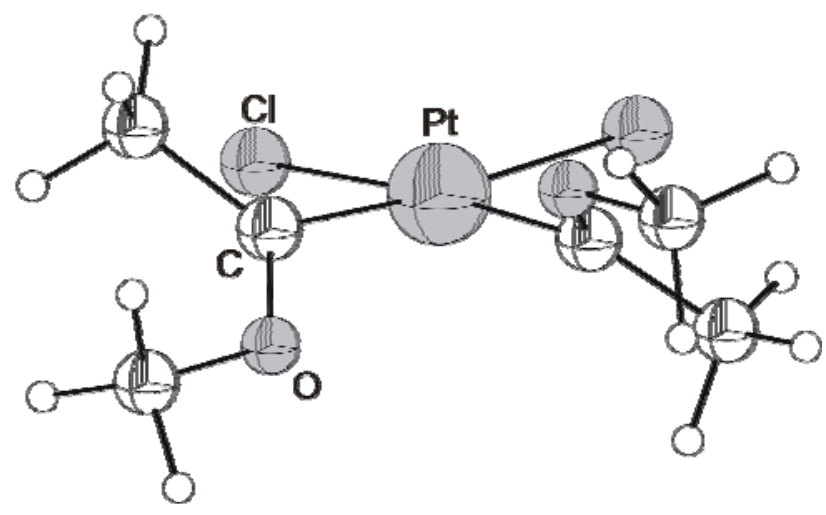

\begin{tabular}{lll}
\hline interplanar angle $^{\text {a) }}$ & $43.6^{\circ}$ & $47.3^{\circ}$ \\
$\mathrm{Pt}-\mathrm{Cl}$ & $2.401 \AA$ & $2.411 \AA$ \\
$\mathrm{Pt}-\mathrm{C}$ & $1.952 \AA$ & $1.945 \AA$ \\
$\mathrm{C}-\mathrm{O}$ & $1.314 \AA$ & $1.314 \AA$
\end{tabular}

a) coordination plane $(\mathrm{C}, \mathrm{C}, \mathrm{Cl}, \mathrm{Cl})$ - ligand plane $(\mathrm{C}, \mathrm{C}, \mathrm{O}, \mathrm{C})$

\section{Complex 11 $\mathrm{zz2}$ :}

cis-[PtCl $\left.{ }_{2}\{\mathrm{C}(\mathrm{OMe}) \mathrm{Me})_{2}\right]$ (carbenes: Z,Z-configuration)

file: ptc12carbene40.out

equilibrium structure

$\mathrm{E}(\mathrm{RB}+\mathrm{HF}-\mathrm{LYP})$

Zero-point vibrational energies

Sum of electronic and zero-point energies
$-1426.10778837 \mathrm{Ha}$

$110.91694 \mathrm{kcal} / \mathrm{mol}$

$-1425.931031 \mathrm{Ha}$

$\begin{array}{llll} & x & y & Z \\ \text { Pt } & 0.459092 & -0.002571 & 0.000673 \\ \text { Cl } & 2.136013 & -1.725750 & -0.080480\end{array}$




\begin{tabular}{|c|c|c|c|}
\hline $\mathrm{C}$ & -0.850304 & 1.434157 & -0.074129 \\
\hline $\mathrm{C}$ & -0.875492 & -1.415965 & 0.077448 \\
\hline $\mathrm{Cl}$ & 2.173296 & 1.683003 & 0.076697 \\
\hline $\mathrm{O}$ & -1.920148 & 1.370242 & 0.688462 \\
\hline $\mathrm{C}$ & -0.722521 & 2.625554 & -0.975193 \\
\hline $\mathrm{O}$ & -1.935273 & -1.344371 & -0.697603 \\
\hline $\mathrm{C}$ & -0.774775 & -2.598978 & 0.992961 \\
\hline $\mathrm{C}$ & -2.930094 & 2.409694 & 0.713205 \\
\hline $\mathrm{H}$ & -1.612027 & 2.708532 & -1.615775 \\
\hline $\mathrm{H}$ & 0.172727 & 2.542759 & -1.586166 \\
\hline $\mathrm{H}$ & -0.647550 & 3.548821 & -0.384629 \\
\hline $\mathrm{H}$ & -0.706251 & -3.529814 & 0.413729 \\
\hline $\mathrm{H}$ & -1.672266 & -2.662035 & 1.624659 \\
\hline $\mathrm{H}$ & 0.114645 & -2.521726 & 1.613146 \\
\hline $\mathrm{H}$ & -3.641327 & 2.092571 & 1.474598 \\
\hline $\mathrm{H}$ & -3.425215 & 2.485889 & -0.258166 \\
\hline $\mathrm{H}$ & -2.485452 & 3.367902 & 0.990211 \\
\hline $\mathrm{C}$ & -2.959111 & -2.370240 & -0.724758 \\
\hline $\mathrm{H}$ & -3.466340 & -2.430832 & 0.241429 \\
\hline $\mathrm{H}$ & -3.657041 & -2.050430 & -1.497235 \\
\hline $\mathrm{H}$ & -2.524211 & -3.336747 & -0.988033 \\
\hline
\end{tabular}

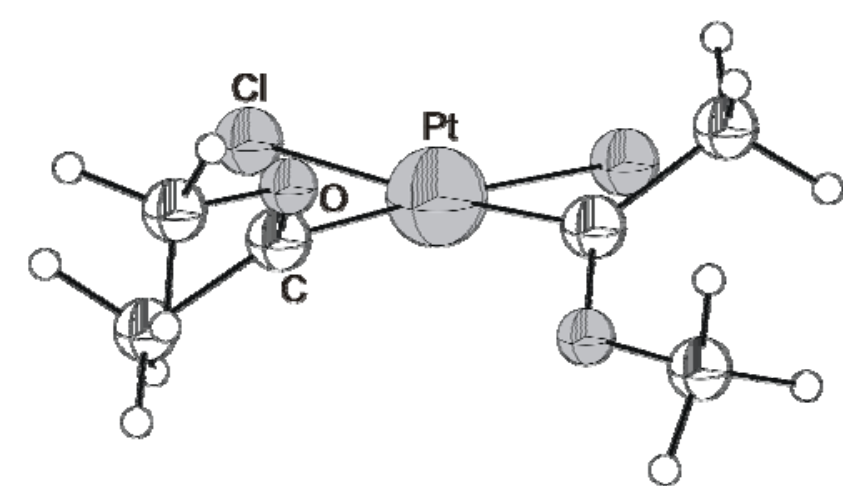




\begin{tabular}{lll}
\hline interplanar angle $^{\text {a) }}$ & $45.1^{\circ}$ & $44.1^{\circ}$ \\
$\mathrm{Pt}-\mathrm{Cl}$ & $2.406 \AA$ & $2.405 \AA$ \\
$\mathrm{Pt}-\mathrm{C}$ & $1.945 \AA$ & $1.945 \AA$ \\
$\mathrm{C}-\mathrm{O}$ & $1.315 \AA$ & $1.315 \AA$ \\
\hline \multicolumn{2}{c}{ a) coordination plane $(\mathrm{C}, \mathrm{C}, \mathrm{Cl}, \mathrm{Cl})$ - ligand plane $(\mathrm{C}, \mathrm{C}, \mathrm{O}, \mathrm{C})$}
\end{tabular}

